\title{
Guidance for Contained Field Trials of Vector Mosquitoes Engineered to Contain a Gene Drive System: Recommendations of a Scientific Working Group*
}

\section{OBJECTIVE}

$\mathbf{T}$ HE FOLLOWING RECOMMENDATIONS represent the response of a group of involved scientists to the need for guidance to aid researchers, government authorities, and community leaders as they consider the design and implementation of field trials to assess the safety and efficacy of genetic strategies for reducing the transmission of diseases by mosquito vectors. Guidance is provided for contained (caged) field trials of genetically-engineered (GE) vector mosquitoes that are fertile and contain novel genetic constructs designed to spread through natural mosquito populations ("gene drive systems"). An effort is made to raise the practical issues that must be considered in advance of such testing, provide generalized recommendations based on currently available information, and identify "points to consider" regarding additional information that may be required in order to make informed decisions on a case-by-case basis. This guidance is intended to clarify the pathway for further assessment of the potential utility of such GE mosquitoes as a tool for the improvement of public health in disease-endemic regions.

\section{FOCUS}

These recommendations build upon similar deliberations for other GE organisms, including transgenic plants and transgenic organisms developed for plant protection; however, they extend previous guidance by addressing directly the principles and practical considerations relevant to development of genetic strategies to control mosquito-borne diseases. It is recognized that the ultimate design of any field trial to assess the safety and efficacy of GE mosquitoes for control of disease transmission will be situation specific and subject to approval of the stakeholders (including communities and government authorities) representing the trial site. Therefore, these recommendations cannot address specifically every detail likely to arise in the planning of a contained field trial, but rather are intended to provide general guidance only, to be taken into consideration as appropriate in the planning process for proposed trials.

Recognizing the multiple genetic engineering strategies that currently are being investigated (Gould and Schliekelman 2004), the number of potential vector species that might be targeted,

*The Core Working Group on Guidance for Contained Field Trials consisted of (in alphabetical order): Mark Benedict, Peter D'Abbs, Stephen Dobson, Michael Gottlieb, Laura Harrington, Stephen Higgs, Anthony James, Stephanie James, Bart Knols, James Lavery, Scott O'Neill, Thomas Scott, Willem Takken, and Yeya Toure. The following Working Group members provided review and comment on the document at several stages of development: Luke Alphey, Kathryn Aultman, Austin Burt, Adriana Costero, Chris Curtis, Heather Ferguson, John Githure, Tom Miller, John Peloquin, Janine Ramsey-Willoquet, Scott Ritchie, Bob Rose, Walter Tabachnick, Ned Walker, and Guiyun Yan. This represents a collective effort of the Working Group members, and the views of all individual members could not be fully represented in this consensus document. Recognizing that the issues addressed herein are complex, and that there are many possible genetic engineering approaches that might be covered by this guidance, it is understood that specific issues will need to be addressed further when field containment plans are developed. The WG members all agree that this document provides useful guidance that should play a primary role in the development of project plans. 
and the number of venues being considered, this guidance is purposely selective in focus. It is intended to address only the earliest stage of field testing of a specific genetic engineering approach ("population replacement") that might be perceived as being the most complex, novel, and challenging. Specifically, this guidance is directed toward assessment of:

- Strategies aimed at developing mosquitoes that are: 1) genetically engineered to have a reduced ability to transmit a particular disease-causing agent (pathogen), and 2) fertile and able to propagate and perpetuate the inheritable trait of reduced pathogen transmission within the indigenous, conspecific, mosquito vector population

The "population replacement" strategy, having the goal of long-term reduction of vector competence, will require a "gene drive" mechanism to spread the effect through the resident mosquito population in a timely way (Braig and Yan 2002, Sinkins and Gould 2006). The recommendations provided here are an effort to proactively address the considerations involved in preparing for and conducting contained field trials for this particular GE approach, and aim for absolute containment. It should be noted, however, that testing of genetic strategies that do not meet the above criteria (such as those seeking to create sterile mosquitoes or genetic modifications that lack a propagative mechanism) may not require the same level of stringency. Nonetheless, it is hoped that the thorough discussion of containment and field trial considerations provided here, including the concept of phased testing, will be broadly useful to inform the planning process for other strategies.

\section{- Contained field trials of uninfected} GE mosquitoes

These recommendations are not intended to address requirements for containment of mosquitoes infected experimentally with human pathogens; however, the type of studies considered here, if successful, may be expected to progress to contained trials of mosquitoes carrying pathogens, and in the future it may prove valuable to extend this guidance to include that possibility.
Additionally, whereas some of the concepts discussed here may be relevant to future consideration of field release trials, this guidance does not address open release experiments or trials. It is assumed that any decision to move beyond contained trials at the field site would require further risk assessment taking into account the information collected from initial contained trials.

This guidance does not deal specifically with issues related to transportation or importation of GE mosquitoes, as these are considered to be covered by existing guidance and regulations, although it is acknowledged that these also must be addressed in the conduct of the field trial.

Finally, this guidance document is not specifically directed toward needs for capacity building in the context of preparation for or conduct of contained field trials, although the Working Group is fully supportive of the importance of this aspect and agrees that it should be addressed to the fullest extent feasible in all facets of the research, planning, and implementation.

\section{DEFINITIONS}

Specific terms may have a different meaning depending upon the context in which they are used. Terms are defined here so that their meaning within this guidance may be readily understood by readers from diverse backgrounds.

Cage or Caged refers to conditions where organisms, GE or otherwise, are physically segregated from the field. Fully-caged conditions that closely simulate natural habitats can serve as an intermediate research stage between controlled laboratory conditions and field trials involving release to the environment. Elements of cage design and procedures for conducting contained field trials are described in Section 7.

Community refers to those people who consider themselves to belong to the area directly affected by a planned trial, and to the informal and formal institutions linking those people with one another, such as village or town councils or voluntary organizations. Community 
engagement refers to activities and processes undertaken by or on behalf of those conducting the field trial and involving residents or representatives of the community, with a view to negotiating mutually acceptable terms and conditions for the conduct of the trial.

Containment refers to those practices that prevent unplanned or uncontrolled release of organisms, GE or otherwise, into the field. This is accomplished by good work practices, strict adherence to standard operating procedures (SOPs) and training of personnel, as well as physical containment within an enclosed structure (referred to in this guidance document as a cage or containment facility). The physical containment afforded by cages may be augmented by biological containment (the use of organisms that have reduced ability to survive or reproduce in the environment) and/or ecological containment (geographic, climatic, or spatial isolation), which limit the spread of organisms into the environment.

Environmental Impact Assessment (EIA) refers to a systematic environmental review process that can serve as a tool for risk assessment. The EIA typically contains a description of the purpose of and need for the proposed action (e.g., the contained field trial), the alternatives to the proposed action, the affected environment, and the possible environmental consequences. This process may also be called a Strategic Environmental Assessment, or Environmental Impact Statement, according to the country of use, but the purpose, intent, and contents are similar.

Field refers to the open environment (not contained) in a study area.

Gene is a segment of DNA that contains information required by cells for synthesis of a product. Related terms are Genome (the full set of genes of an individual) and Genotype (the genetic constitution of an organism).

Gene drive refers to a mechanism that spreads the desired gene and its effect (e.g., inability to transmit a pathogen) into the resident mosquito population in a timeframe that is appropriate for disease control.
Horizontal gene transfer refers to the heritable transfer of a functional genetic element from one organism to another without mating. This term is applied most often to genetic exchange between species.

Genetically-engineered (GE) organism refers to any organism that has in its genome novel DNA of endogenous, exogenous, or mixed origin that was made using modern recombinant DNA technology (also termed transgenic DNA). Although successive selective breeding of strains of organisms with naturally-occurring allelic variations also results in geneticallymodified strains, these are excluded from this definition.

Transgenic and transformed also are used interchangeably here to refer to genetically-engineered organisms. Other terms sometimes used interchangeably include geneticallymodified organism (GMO) or living modified organism (LMO).

Harm is used to describe a detrimental outcome of an event or activity. In the case of GE mosquitoes, possible undesirable consequences affecting public health, the environment/ecosystem, the economy, or other aspects of the "quality of life" may constitute harm.

Adverse effect is used to denote a molecular, biochemical or functional change ultimately leading to harm.

Hazard is an event, condition, or activity with the potential to cause harm. An example of hazard is the induction of a new and undesirable phenotype in the target species, such as increased vector competence or vectorial capacity. "Hazard" and "risk" are two terms that are often used interchangeably; however, the relationship between hazard and risk is not necessarily so direct (see "Risk" below). Hazard has been further categorized (Fiksel and Covello 1986) as:

Probabilistic if the hazard exists and has occurred at least once. An example would be airplane crashes, where a frequency of occurrence can be calculated and the risk can be quantified based on prior crash data. Hypothetical if the hazard has not been known to occur but there is a scientific line 
of reasoning to support its potential existence. A property of hypothetical hazards is that specific hypothesis-testing models or scenarios can be developed. An example would be the development of an antibiotic resistant pathogen as a result of horizontal gene transfer from plants to microbes. The transfer of plant genes and their stable integration within the genome of bacteria present in either the gut or soil has not yet been observed in nature. Nonetheless, based on our knowledge of molecular biology and observations of gene transfer among bacteria, we can hypothesize that it might occur between plants and microbes and can estimate an approximate likelihood based on the stepwise probability of individual preconditions. Speculative if the hazard has not been known to occur and there is no reasonable scientific line of reasoning to suggest that it will occur in the future. Speculative hazards are not generally amenable to rigorous hypothesis testing.

Modeling is an attempt to describe the behavior of a natural system or to predict the likelihood of an event occurring within that system, and may utilize mathematical formulas and computer simulations.

Pathogen is an organism that causes disease. In dengue infection, the pathogen is a virus, whereas in malaria, the pathogen is a unicellular parasite.

Phenotype refers to the observable characteristics of an organism, based on genetic and environmental influences.

Population reduction refers to methods that decrease the overall number of vectors in a region. These methods can be specific to a particular type of vector, or nonspecific, and may include chemical (e.g., insecticides), biological, environmental, mechanical, or genetic approaches.

Population replacement refers to methods that change the genetic make-up of a vector population; as envisioned here, the change would result in decreased ability to transmit a pathogen. This is anticipated to be achieved by using genetic engineering to introduce sequences (or a gene/genes) that reduce vector competence in the context of a gene drive system that will cause the frequency of the gene to increase within the population to a level where it has an observable impact on disease incidence.

Public engagement refers to interaction with the wider society of which the community of interest is a part.

Regulations are considered to include all statutory requirements, whether in the form of laws, regulations, covenants, treaties, or other legal instruments, that must be complied with before commencing field research with GE mosquitoes.

Risk is the likelihood, or probability, that harm will occur. The level of risk is estimated as the product of the expected probability (likelihood) that a harmful event will occur and the expected consequences (impact) of that event. Qualitatively, likelihood can be rated from very unlikely to very likely and impact from negligible to critical. For example, where it is considered that horizontal transfer could be hazardous for nontarget species, comparisons of two genetic constructs with differing capabilities for horizontal transfer (i.e., low vs. high) can be described as representing the same hazard, but the risk associated with the latter is greater.

- Risk assessment is a systematic and scientific evaluation of the level of risk for any hazard. The product of risk assessment is a statement regarding the probability that harm will occur.

- Risk management refers to strategies that are developed to avoid and reduce risk, or to accept the consequences of the risk.

- Perceived risk refers to the perceptions of risk attributed to a proposed or existing trial. Perceived risk may be informed by risk assessment, but it may be influenced additionally by social or cultural factors, such as religious views or lack of trust towards regulatory bodies. Different individuals or groups may differ in their per- 
ception of risk, even for the same trial and risk assessment data.

Standard Operating Procedures (SOPs) are written instructions that describe in detail the routine practices to be followed for a specific operation, and are intended to ensure that the task is performed correctly, consistently, and safely by anyone who performs it.

Transposable elements, or transposons, are discrete pieces of genetic material (DNA) that can move from one location in the genome to another. This process is referred to as transposition. Transposable elements may be constructed to carry other genes with them, as a tool for genetic engineering.

Vector mosquitoes are those mosquitoes that are able to transmit a disease-causing pathogen to people or animals. The vectors of dengue and yellow fever viruses (Aedes species), and those of human malaria parasites (Anopheles species) are used as examples in these guidelines.

- Vector competence is the genetic capability of a mosquito to serve as a host for the complete development and/or replication of a specific pathogen (Hardy et al. 1983).

- Vectorial capacity is a numerical index of the potential for a mosquito population to transmit a pathogen, and includes vector competence, feeding preferences, longevity, etc. (Garrett-Jones 1969).

\section{BACKGROUND AND RATIONALE}

The concept of genetic control of arthropod vectors of disease is not new, and considerable research to apply this concept to mosquitoes was conducted during the 1970s using technology available at that time (e.g., Curtis et al. 1976). Substantial scientific progress has been achieved since early hypotheses that the application of modern genetic engineering technology to mosquitoes could provide a useful approach for limiting transmission of pathogens causing diseases such as dengue and malaria (e.g., Collins and James 1996). This has led researchers to evaluate the feasibility of entering into initial testing of GE mosquitoes in the foreseeable future. Laboratory-based discovery research is now underway on a variety of genetic engineering strategies for controlling arthropod vectors of disease (Braig and Yan 2002, Sinkins and Gould 2006), with the long-range goal of eventual implementation to improve public health in disease endemic regions.

Extensive experience exists for using insects altered by exposure to radiation or chemical mutagens to control agricultural and livestock pests, and no untoward consequences have been reported. However, researchers pursuing a genetic engineering approach to the control of vector-borne diseases, and their supporting organizations, recognize that no similar experience exists, and that each new strategy must be advanced through careful and systematic procedures, with safety for individuals, communities, and the environment as the highest priority (Toure et al. 2002). Moreover, they are cognizant that the public expects particular caution in the testing of strategies employing genetic engineering. Therefore, it has been proposed that such testing should take place in a logical and stepwise manner, analogous to the well-accepted practices applied to clinical trials in the development of other prevention/intervention methods such as drugs and vaccines, with appropriate monitoring of safety at each step. Implementation of containment methods can serve to improve safety conditions and reduce risk in early trials of GE mosquitoes.

\subsection{Containment Considerations}

Determination of the level of risk associated with the use of transgenic or GE organisms must take several variables into account; these include the potential for inadvertent spread of the novel genotype within or between species, the nature of any unintended consequences that might result, and the extent of such consequences. Risk will be reduced by measures or conditions that lower the potential for undesirable consequences. One way to reduce risk is to limit the size/scale of the planned activity to the extent practicable for achieving meaningful results. Containment methods can also contribute to risk reduction. Such contained 
testing should be designed to evaluate sciencebased hypotheses that will inform subsequent decisions on larger scale and on nonconfined field tests. For example, risk of dispersal and establishment in the field would be lower if the GE organisms are sexually sterile or if the transgenic material is intrinsically unable to propagate or is functionally inactive in any but the intended setting. The utility of building such safeguards into the transgenic organism as a form of biological containment will differ according to the control strategy being pursued (e.g., population reduction vs. population replacement). The GE mosquito strategy under consideration here ultimately aims for population replacement, and therefore, although it may help to reduce risk in the early stages of testing, biological containment is inappropriate in later stages of research addressing questions of safety and efficacy.

Ecological containment offers another method for reducing risk, in which studies are conducted in an environment that is inhospitable (e.g, because of climate) to the survival or spread of the GE mosquito. However, there also may be undesirable aspects to conducting trials in inhospitable environments if this involves testing conditions that are artificial or the introduction of vector mosquitoes outside their natural range. Furthermore, the ethical principle that an individual (or community) that cannot benefit from the research should not be subjected to the risks may be applicable in this situation.

Physical containment, which intends to keep the transgenic organisms segregated from the open environment, provides another method for decreasing risk by limiting the potential for spread. Cages provide a form of physical containment in the context of field testing (Knols et al. 2002) and have been advocated by experts for use in the early stages of testing GE mosquitoes (Alphey et al. 2002, Scott et al. 2002). A trial conducted within outdoor cages that are exposed to the ambient environment in a region where the strategy might ultimately be applied for the control of vector-borne disease will allow researchers to study how GE mosquitoes might survive and compete under more natural conditions of climate and light variation, in the presence of local wild-type mosquitoes, etc., while separating the GE mosquitoes and their genetic material from the open environment (Fig. 1).

This Working Group agrees that initial testing of transgenic mosquitoes beyond the laboratory should be conducted under confined conditions to minimize any possibility of escape into the environment. The guidance provided here addresses specific needs and considerations for the conduct of contained field trials using GE mosquitoes as defined in Section 2, focusing on the physical containment provided by outdoor cages and including ways

\section{Benefits of Contained Field Trials}

- Simulate performance in natural environment

- climate variation

- light variation

- spatial and structural similarity

- natural behavioral interactions

- Provide more consistent quality data than open field studies

- limited dispersal increases chance of recovery

- experimental conditions can be manipulated more readily

- number, sex, age and physiological status of released insects can be controlled

- Increased safety

- limited dispersal minimizes numbers of GE mosquitoes required

- allows separation from open environment 
in which that may be enhanced by other containment methods and verified by standardized monitoring procedures. In this regard, it may also be helpful to refer to similar containment recommendations that have been developed for transgenic plants, microbes, and plant pests. $^{+}$

\section{RECOMMENDATIONS FOR PHASED TESTING}

GE strategies that show sufficient promise in laboratory-based discovery and development research will proceed to further testing for safety, efficacy, and acceptability. Caged testing will be conducted first within the laboratory under the appropriate laboratory containment, as specified elsewhere (e.g., Arthropod Containment Guidelines 2003). However, a setting more closely representative of real-world conditions will ultimately allow better prediction of the effectiveness of the strategy. This section provides recommendations for a structured, stepwise, testing process for transgenic mosquitoes that emphasizes safety and risk management.

Acceptance criteria for progression from one phase of testing to the next should be established by those conducting the trial in agreement with relevant authorities as appropriate. The specific criteria are likely to vary according to the nature of the genetic approach being studied and will reflect the points to consider for risk assessment described below (Section 6.3), but generally should take the following into account:

- Were the performance and results in the previous phase within acceptable limits?

${ }^{+}$Traynor, PL, Adair, D, Irwin, R. A Practical Guide to Containment: Greenhouse Research with Transgenic Plants and Microbes. Blacksburg, VA: Information Systems Biology; 2001. www.isb.vt.edu/cfdocs/greenhousemanual. $\mathrm{cfm}$

NAPPO Regional Standards for Phytosanitary Measures, RSPM No. 27, Guidelines for Importation and Confined Release of Transgenic Arthropods in NAPPO Member Countries. Ottawa, CN: North American Plant Protection Organization; 2007. www.nappo.org/ Standards/Consultation/RSPM27/RSPM\%2027-Version March\%205\%202007-e.pdf
- Were the safety measures at the previous phase of testing sufficient?

- Is additional information needed before proceeding to the next phase?

- What experimental or procedural modifications are necessary before proceeding?

\subsection{LABORATORY STUDIES}

The laboratory phase will integrate biological studies, in which the basic molecular, genotypic, physiological, and behavioral characteristics of the transgenic mosquito will be evaluated and compared with those of wildtype mosquitoes in order to characterize its phenotype, with subsequent development of analytic or computer simulation models to facilitate prediction of the potential effects under anticipated field conditions. This phase should include not only assessment of the expected beneficial effects, but also intensive examination of the potential for adverse effects, both in target mosquitoes (either the transgenic mosquitoes or in others belonging to the same species, termed conspecific) and in nontarget species.

\subsubsection{Biological studies}

Whereas studies of transgene effects may be carried out initially in a laboratory strain of mosquito, the next logical step is to introduce the gene of interest into mosquitoes derived from the location where field trials are contemplated. Introduction of the gene into wildtype mosquitoes could be performed by independent germline transformation or genetic crosses. The biological assessment will include determination of genotype and phenotype of offspring proximal to the transformation event or cross, as well as, to the extent feasible in the laboratory, evaluation of the stability of these characteristics in subsequent generations. Modeling studies (Section 5.1.2.) will additionally contribute to prediction of long-term effects in target mosquitoes.

These studies should be conducted under accepted guidelines for laboratory research (e.g., Arthropod Containment Guidelines, 2003, www.liebertonline.com/toc/vbz/3/2, or Guidelines for Certification of a Physical Containment Level 2 Arthropod Facility, www.ogtr. 
gov.au/rtf/certification/PC2ARTHv2-1.rtf), and include:

- Assessing the effect on the ability of target mosquitoes to transmit the pathogen of interest

- Evaluating the ability of the genetic engineering strategy to spread the transgenic DNA through a mixed population of transgenic and wild-type mosquitoes of same species

- Testing for unintentional or adverse effects on the target mosquitoes, which may include:

- increase in vectorial capacity or vector competence for nontarget pathogens (especially those transmitted at the potential field site)

○ increase in blood-feeding

- alteration of feeding behavior and/or oviposition (egg-laying) behavior

- alteration of population dynamics (e.g., potential changes in sex ratio, increased longevity, population growth rates, carrying capacity that can be supported by the environment)

- alteration of the immunogenic profile of salivary gland proteins

- decreased susceptibility to control or surveillance measures (e.g., insecticides, attractants)

- Testing of persistence of the transgene in the environment (e.g., soil)

- Testing of the potential for horizontal gene transfer between transgenic mosquitoes and a representative subset of organisms.

Investigators should have in mind a specific set of performance characteristics that must be met in the laboratory to justify proceeding to contained field trials. The Working Group recommends that the following observations in the laboratory should preclude a GE mosquito from being considered for further study in cage trials: 1) increased vector competence for the pathogen under study or other pathogens present at the field site; 2) a changed biology making it more difficult to control; or 3) horizontal gene flow to nontarget organisms likely to have undesirable ecological or environmental implications.
Horizontal gene transfer (HGT) is a major concern in the use of gene drive systems to both the scientific and nonscientific community. The most significant component of this concern is the potential for negative impact on a nontarget organism. The Ecological Society of America has evaluated the possible ecological effects of GE organisms, including those associated with horizontal gene flow (Snow et al. 2005). Their report states that the effects of GE organisms will depend on a variety of biological, physiological, and geographical factors, and confirms the need for a case-by-case analysis of ecological effects that integrates laboratory and field studies and includes data on spatial and temporal variability. A major recommendation of this report is the early involvement of ecologists, evolutionary biologists, and other specialists, in the design and conduct of studies aimed at quantifying the benefits and risks posed by GE organisms in the environment. In this regard, the Working Group recommends that for each particular GE approach under investigation, a systematic and multidisciplinary approach be applied to evaluation of the likelihood of HGT to cause a negative impact on nontarget organisms. This systematic approach should address two questions: 1) what is the probability that the transgenic DNA (effector construct and/or specific proposed gene drive system) will move out of its intended target to another species, and 2) if it does move, what are the possible outcomes? For example, in studies of the potential for HGT between transgenic plants and microorganisms, methodologies have been developed to respond to the first question and selective pressure has been recognized as a strong influence on the second (e.g., Bertolla and Simonet 1999). The evaluation process used in other systems includes the following steps: 1 ) identify potential organisms that would most likely be the recipients of HGT (primary recipients are expected to be almost exclusively bacteria and viruses), 2) establish circumstances in the laboratory favorable to HGT (for example, in bacteria, organism density and energy state (nutrition) are maximized), and if possible, 3) define a frequency for HGT. Once these are determined, 4) the proposed field site should be evaluated for conditions that approach those identified in the lab- 
oratory as favorable for HGT. The degree to which these conditions are present will provide a way to estimate the probability that HGT will occur in the field. These probabilities then can be used as a basis for decision-making about whether to undertake cage trials of the specific approach under investigation.

Implementing the systematic, multidisciplinary approach recommended above will depend on recruitment of outside consultants not only to complement the expertise of the research team, but to assure a level of authority and transparency to the evaluation. Consultants should include experts with the appropriate understanding of relevant ecological and evolutionary processes to aid the research team in selection of nontarget organisms for testing, as well as design and interpretation of experiments necessary to address the four steps in the evaluation process. Consultants should provide scientifically-defensible recommendations to be used by researchers, funders, and other stakeholders in decision-making about progression to cage trials. These consultants should be recruited early enough in the development process to provide advice on design of the GE strategy so as to minimize the potential for environmental risks, and they should remain with the project through cage trials in order to evaluate the ecological effects of the GE mosquito in the intended ecosystem.

\subsubsection{Modeling studies}

Using information obtained from biological studies and previous knowledge from studies of the epidemiology and natural history of the disease, this phase of testing may include mathematical and computer simulation modeling of the expected results of the proposed contained field experiments, including the probable spread of the transgene, mutation rates, and the effects on the phenotypic profile of the local mosquito population. Modeling may also assist in evaluating the impact of the gene drive system, the insertion events and/or the inserted genetic material on the genome of the target population over several generations, and predicting the possible range of future effects. Information obtained from such studies should contribute to the formulation of acceptance criteria for moving to contained field trials.
Modeling also may contribute to risk management by helping the investigators predict results of an inadvertent release of transgenic material at the location of the proposed contained field trial. This should include considerations of the intrinsic mobility of the transgene, consequences of mutation, and its fitness consequences (such as its effect on the mosquitoes' ability to survive and reproduce in the environment). In this context, modeling also may provide valuable insights into the requirements for an effective remediation strategy.

An iterative loop is envisaged, in which new information obtained from contained field trials (e.g., improved fitness parameters obtained from assays under more natural conditions) will allow for the further improvement of models, which can then be used in subsequent trials and will provide increasingly field-comparable predictions.

\subsection{The Project Plan}

Results of the biological and modeling studies will be used to prepare the preliminary plan for field testing (herein referred to as the Project Plan). The Project Plan will be used to describe the project in interactions with the institutional and/or national bodies having responsibility for overseeing and regulating such trials. Its initial creation is the last step of the laboratory phase. The Project Plan should be revised as required by the responsible authorities, and updated throughout the various phases of testing as new data are collected and protocols refined.

The Project Plan should provide a clear description of the scientific rationale for entering into the next phase of testing, the experimental design proposed for contained field trials, and the precautions to be taken. The plan may be supplemented by detailed protocols, SOPs, and/or an Environmental Impact Assessment (Section 8.5) according to the requirements of relevant authorities. The Project Plan should include:

- The evidence for the proposed efforts to interrupt pathogen transmission, and an explanation of the potential for reducing disease incidence 
- A preliminary risk assessment of the potential for harm based on the results of laboratory studies, including background information on the genetic elements involved in the transformation (transgenic DNA, genetic drive mechanism, and, if available, findings of analogous experiments in other organisms) and a plan for assessing the potential for harm in the caged field trial

- Preliminary evidence from laboratory and modeling studies justifying the claim of the lack of harm in the event of accidental escape of a GE mosquito or its genetic material

- A detailed organizational structure for the project, defining administrative responsibilities and delineating the decision making process for all components (Section 7.1)

- Specific plans for the first phase of the activities to be performed under contained field conditions, including plans for risk management (including methods for containment, monitoring, and remediation, as well as plans for safety and security oversight)

- Plans for community engagement, including risk communication.

\subsection{Contained Field Trials}

This phase initiates contained field trials whose design and safety have been approved by the relevant institutional bodies and regulatory authorities (Section 8). Experimental protocols for contained field trials should be designed carefully to maximize safety and provide a reasonable expectation that results will yield meaningful insights into the potential utility of the experimental strategy.

In some cases before moving to a field site in a disease-endemic region, it may be necessary to perform initial contained field trials in an environment that is only transiently suitable for survival of the GE mosquitoes under study (a method of ecological containment). This decision, which must be based on scientific, economic, or biosafety concerns, would be made by agreement of the investigators conducting the trial and the relevant institutional and national bodies.

Trials to be conducted under environmentally and epidemiologically realistic conditions (as in a disease-endemic region) relative to eventual implementation for disease control must consider hazards qualitatively similar to those of an actual field release. The major difference is that under containment the risks associated with these hazards are reduced greatly. Consultation with ecologists and evolutionary biologists at this phase will aid in the design of studies that predict interactions with the actual ecosystem to the fullest extent possible under caged conditions, and will be important for decision making regarding any eventual progression to open field release trials.

\subsubsection{Pretrial testing}

This phase will begin even before the containment facility is constructed, at the time of site selection, with initiation of epidemiologic, ecologic, and social science studies (Section 9). After cages are designed and built, the safety of the facility and the procedures under which transgenic material will be handled must be established prior to the initiation of trials. Safety and quality assurance experiments using nontransgenic local mosquitoes will therefore precede the actual trial. Any findings resulting from this pretrial phase will be included as a modification of the Project Plan. Studies conducted during this phase may include:

- Collecting baseline epidemiological information in the vicinity of the proposed experiments, particularly assessment of the disease targeted for reduction and other vector-borne diseases whose transmission reasonably could be expected to be affected by inadvertent release of the GE mosquitoes

- Evaluating mosquito ecology and population genetics at the proposed site

- Establishing suitable conditions for maintenance of local wild-caught mosquitoes within outdoor cages using the protocols and containment measures described in the Project Plan

- Assessing the ability of the structure and procedures to prevent escapes and breach of containment

- Testing the procedures to be implemented for prevention of inadvertent release (see Section 7.4.1.) 
- Testing the measures to be used to detect escape of contained transgenic mosquitoes (Deliberate release of marked, locallyderived, nontransgenic mosquitoes $\ddagger$ at the location of the containment facility can be useful to determine the sensitivity of the proposed measures for detecting escape.)

- Testing the effectiveness of external measures (e.g., vegetation-free zones, traps, etc.) intended to prevent dispersal of inadvertentlyreleased material via mark-release-recapture

- Testing the proposed remediation strategies to be used in the event of escape as appropriate (Mark-release-recapture strategies should be considered for this purpose.)

- Conducting social science studies in the vicinity of the proposed experiments with a view toward identifying the nature and determinants of perceived risks and other factors that might influence the acceptability of any proposed trial, and to developing on the basis of these studies a strategy for authentic engagement and negotiation with the host community.

\subsubsection{Contained field trial: Biosafety and experimentation}

Before this phase commences, the investigators and all relevant authorities should review the results of the pretrial biosafety and quality assurance tests and recommend any necessary modifications to the Project Plan. After the revised Project Plan receives all necessary approvals, experiments with uninfected transgenic mosquitoes may begin.

\subsubsection{Unmixed transgenic population}

Some transgenic mosquito strains may need to be evaluated in the absence of wild mosquitoes in the containment facility. Studies will ex-

\footnotetext{
$\ddagger$ In mark-release-recapture studies, a known number of clean, disease-free mosquitoes that have been marked in some way, such as by dusting with fluorescent dye, are released in an area. Traps are placed in the vicinity, and, after a period of time, the mosquitoes caught in the traps are examined for markings. Recapture rates will need to be indexed appropriately to provide an estimate of the efficiency of traps for detecting escaped GE mosquitoes.
}

amine genetic and genomic stability and determine whether the transgenic strain genotype or phenotype change unexpectedly when measured under contained field conditions. Such studies include:

- Determining the viability and life-span of transgenic mosquitoes under the conditions determined suitable for the maintenance of wild mosquitoes

- Determining whether the genotype or phenotype (including behavioral characteristics and interaction with the vertebrate host) of the transgenic strain differs in comparison to laboratory predictions (see Section 5.1.1.)

- Frequent monitoring of external traps and other mosquito collection methods for the presence of GE mosquitoes

- Frequent monitoring inside and outside the cage for the presence of transgenic DNA in arthropod predators, vertebrate insectivores, vertebrates serving as blood hosts for the study, and other nontarget organisms found in the vicinity (according to the advice of consultants, see Section 5.1.1.)

\subsubsection{Mixed transgenic and wild populations}

Findings and any resultant modifications in practices or procedures resulting from any previous testing of the transgenic population should be included in the final Project Plan to be reviewed by the appropriate authorities. Upon obtaining approvals, native local mosquitoes may be introduced into the containment facility to conduct longer term studies that assess the ability of GE mosquitoes to compete for and mate with wild mosquitoes within the containment facility. It is anticipated that these interactions will be assessed over the course of several mosquito generations in order to obtain a meaningful understanding of the effects, but the length of these studies may vary according to the GE strategy under consideration and should be specified within the Project Plan. During this phase, assessment of genomic, genotypic, and phenotypic changes in target and nontarget species will be conducted on a continuing basis. 


\section{CONSIDERATION OF HAZARDS AND RISKS}

The ultimate motivation for developing transgenic mosquitoes is their eventual release for beneficial purposes - that is, reduction of the transmission of specific pathogens causing diseases responsible for substantial human suffering. In considering how GE mosquitoes might cause harm, questions about possible effects on public health, the environment/ ecosystem, agriculture, the economy, or other aspects of the "quality of life" have been raised. It is important to note that risks associated with testing of GE vectors must be balanced against the potential benefit of reduction in disease incidence. This balance is best taken into account by the countries and communities that are considering hosting the field trials and/or eventually implementing the control strategy, and where the diseases in question may be of immediate concern.

In laying the groundwork for contained field trials, the principles of risk assessment dictate that hazards associated with the use of GE mosquitoes be identified. For those hazards that are identified, the likelihood that harm may result (risk) then is considered. If it is determined that the likelihood of a hazard occurring, or the possible negative consequences should it occur, are so small as to be essentially negligible, the hazard could be considered to pose no significant risk.

Risk assessment in the preparation for trials of GE vector mosquitoes must be based on state-of-the-art scientific understanding as well as common sense. Common sense dictates that if the transgenic material is successfully kept separate from the external environment, the probability that harm will occur is essentially zero. In the judgment of this Working Group, no risk is posed under circumstances in which the GE mosquitoes remain isolated physically so that neither they nor their genetic material have direct interaction with the environment outside of containment.

Common sense also dictates, however, that one must plan for the possibility that containment may fail. Hazards are presented in situations (such as an escape from the cage) that might allow the transgenic mosquitoes or their genetic material to interact with other organisms in the open environment. Whereas it cannot be assumed that the hazards presented by a situation such as an escape will inevitably result in harm, our current understanding is insufficient to rule out this possibility. Therefore, a major goal of this guidance is to identify the possible hazards should transgenic mosquitoes or material under study in contained field trials come into contact with the open environment. This requires consideration of all viable life stages of the GE mosquito (egg, larva, pupa, adult), as well as the genetic material itself, and the possible biological interactions.

Where possible, general recommendations are offered to reduce the hazard and minimize the risk. Mathematical and/or quantitative estimation of risk is currently theoretical due to the paucity of available information. Both the probability and the consequences of an event occurring will vary according to the specific strategy being tested, the specific conditions under which it is being tested, etc. Therefore, points-to-consider have been included as guidance on the kinds of information that may be necessary to conduct meaningful risk assessment on a case-by-case basis.

\subsection{Hazard Categories Unique TO GE ORGANISMS}

Potential concerns related to genetic engineering include, but are not limited to, vertical inheritance, horizontal gene transfer, and gene silencing (Pew Initiative 2004). There are two major categories of hypothetical hazard that must be considered with respect to contained field trials of transgenic mosquitoes, although, as discussed below, these hazards pose different levels of risk.

\subsubsection{Effects on target mosquitoes}

One category is the hypothetical hazard that could be associated with premature transfer of genetic material to free-living wild mosquitoes of the same species as the GE mosquitoes being tested, before the safety and utility of the strategy has been adequately tested. The hazard to be considered is the possibility that genetic engineering to reduce vector competence 
would directly or indirectly alter the function or behavior of target mosquitoes in undesirable ways. Undesirable direct effects could result if the genetic engineering strategy itself has unforeseen and unintended consequences. Undesirable collateral effects might result if an introduced transgene became inserted into, or indirectly modified the function of, other endogenous genes.

Possible adverse effects on target mosquitoes include increased capability to transmit the pathogen under study or other nontarget pathogens in circulation at the field site, changes in fitness that might enhance survival or reproductive capacity, changes in behavior that might increase biting or other nuisance activities, and unintentional genetic or behavioral changes that might decrease susceptibility to control measures. These possibilities must be evaluated as thoroughly as feasible in laboratory studies, including small cage testing, before a decision is made to move forward to contained field trials (see Section 5.1.1.).

It must be understood that undesirable effects on mosquito population dynamics (such as potentially detrimental changes in sex ratio, longevity, population growth rates, or carrying capacity) may not become obvious in the laboratory. Indeed, this is a key reason for conducting contained field trials under conditions that simulate the natural situation as closely as possible, as a transition step between controlled laboratory experiments and field implementation. Distinguishing such effects will require long-term sampling to monitor potential changes in fitness and other key indicators.

\subsubsection{Effects on nontarget organisms}

This hazard category includes those associated with transfer of genetic material to nontarget organisms. Horizontal transfer of transgenic DNA between species could theoretically occur directly or via intermediaries such as viruses or other microbes capable of infecting multiple hosts. Possible adverse effects include:

- Acquisition of a new and undesirable capability (e.g., increased capacity to transmit a pathogen)
- Acquisition of a new and undesirable behavior (e.g., increased blood-feeding frequency, altered host preference)

- Disruption of an essential or beneficial function (e.g., decreased pollination)

- Reproductive effects in a nontarget organism (e.g., sterility, mutations)

- Alteration or disruption of the normal interactions of organisms within the environment (ecosystem effects).

Consideration should be given to developing transgenic constructs that are incapacitated, attenuated, or replication-incompetent in nontarget organisms as a method of reducing risk that might be associated with horizontal transfer. Evaluation of horizontal transfer in laboratory testing (Section 5.1.1.) should provide useful insights for risk reduction. A GE strategy must not be moved forward to contained field trials if laboratory studies revealed horizontal transfer to nontarget organisms occurred and was likely to have undesirable environmental or ecological implications.

Assuming horizontal transfer did not occur in laboratory studies, this may be characterized as a speculative rather than hypothetical hazard. Nonetheless, precautionary monitoring during the trial is recommended, and assay for presence of the transgene is proposed as the principal method (Section 7.2.4.). This should be planned according to the advice of project consultants (Section 5.1.1.), and could include periodic monitoring of nontarget organisms such as other species of mosquitoes, other arthropods, and microbes such as viruses or bacteria. Uptake of genetic information by higher organisms (e.g., vertebrates, plants), while considered less probable from a biological perspective, still must be taken into account when planning monitoring activities.

\subsection{Predisposing Events And Activities}

Having acknowledged that certain hypothetical or speculative hazards are posed by contact of transgenic mosquitoes or their genetic material with the environment outside containment, it is reasonable to begin preparation for contained field trials by considering the conditions under which breaches of 
containment might happen and how these conditions might be avoided. Whereas this guidance document is focused on testing within the field cage, the potential for release during transportation from the production site, if located elsewhere, to the cage must also be acknowledged and preventive plans developed.

\subsubsection{Breach of containment}

A physical breach of the containment facility structure or any subordinate structures (e.g., a field laboratory), whether inadvertent or intentional, could lead to a release of transgenic mosquitoes or genetic material derived from them. In the event of a containment breach, the number of mosquitoes or amount of material that might escape would vary according to the type of breach that occurred, and this would influence proportionally the probability that harm might result.

Structural failure of the containment facility is an obvious potential cause of inadvertent release.

Causes of structural failure include:

- Predictable environmental or weather-related damage and deterioration of structural materials (e.g., wind, rain, UV exposure)

- Unpredictable environmental damage (e.g., tornados, earthquakes, lightning)

- Human damage (e.g., vehicles)

- Animal damage.

Recommendations to minimize the hazards resulting from structural failure include:

- Designing cages to prevent damage from weather or environmental conditions (Section 7)

- Planning experiments so that they are not conducted during "high risk" times (e.g., during a monsoon season)

- Restricting vehicular access and parking around the facility

- Providing SOPs that include plans for frequent routine examination of the integrity of the field cage, along with how to react in the event that damage has occurred (Section 7).
Inadvertent release may also be caused by human error, accidents, or poor planning. Deviations from Project Plan protocols may include:

- An unavoidable accident (e.g., leakage of a water source containing eggs, larvae, and/or pupae)

- A preventable accident (e.g., leaving a door open)

- A conscious relaxation/unapproved change of protocol

- Inadequate education and training of personnel.

Recommendations to minimize hazards resulting from protocol deviations include:

- Planning cage design and placements within the cages to minimize the possibility for accidents

- Providing clear and explicit protocols and SOPs in the language(s) of the employees, and putting a plan for oversight in place (Section 7)

- Rehearsing all procedures during the Pretrial Testing phase

- Developing safety courses and repeating them on a frequent basis.

Deliberate actions, whether intended to disrupt the project or not, also may contribute to breach of containment. Deliberate actions that might result in release include:

- Vandalism or sabotage

- Burglary (to obtain valuable material within the cage).

Recommendations to prevent deliberate release focus on establishment of good community relations/involvement, education, and outreach, which may be the most effective deterrent, but also extend to physical and procedural precautions, including:

- Taking into account in the process of site selection those local, ethical, social, and cultural issues that might influence the acceptability of the trial (Section 9)

- Maintaining an ongoing commitment before, during, and after the trial, to interactions 
with the public, media, and local authorities in order to provide accurate information and to answer questions (Section 9)

- Installing physical barriers and security systems (Section 7)

- Performing background checks on potential employees.

\subsubsection{Other interaction with the environment}

The possibility that mosquitoes successfully contained within the cage might still interact with other organisms also must be considered. Although the likelihood of this presenting a hazard is expected to be low if the containment facilities and procedures are appropriately designed, it will be particularly challenging to monitor this type of interaction.

One possible scenario for this type of interaction would involve transmission through the cage. It is possible that GE mosquitoes residing within a cage could mate with wild local mosquitoes through the mesh of the cage walls. Recommendations for preventing this possibility emphasize planning for cage design that prevents contact with the outside environment via use of two walls of screening (Section 7.1.2.).

An additional possibility involves interactions within the cage. Thus, fully contained GE mosquitoes might interact directly with other organisms inside the cage, posing a hazard only in the case that nontarget organisms somehow carry the transgene back to the open environment at a later time. Other organisms could come into contact with caged mosquitoes by:

- Exposure of microbes, nematodes, etc., resident in soil or on plants within the cage

- Entry of small invertebrate or vertebrate organisms from the open environment (through cracks, mesh, drains, etc.)

- Exposure of employees working within the cage or inadvertent introduction of other organisms by employees on clothing, footwear, tools, equipment, etc.

- Exposure of animals brought inside the cage as a source of bloodmeal or inadvertent introduction of other organisms carried by those animals
- Carriage of microbes into the cage by air, water, soil, etc.

Recommendations for preventing hazards posed by interaction between GE mosquitoes and other organisms within the cage are discussed in Section 7, and include:

- Designing transgenic DNA to be nonfunctional in nontarget organisms

- Designing the containment facility in such a way as to prevent entry/exit of small organisms

- Establishing SOPs for decontaminating soil, plants, structures, etc., before removal from the cage, or safely transporting them to a location at which decontamination can safely be performed

- Establishing SOPs for preventing interactions between mosquitoes and employees within the cage and for decontamination of employee clothing upon exit from the cage

- Establishing SOPs for minimizing contact of vertebrate animals with the cage interior and for decontaminating animals upon exit from the cage

- Establishing SOPs for treatment of drainage water from the cage.

\subsection{Risk Assessment: Points to CONSIDER ${ }^{\S}$}

The intention of phased testing is to limit the risk from a variety of factors that presently are unknown, so that the level of uncertainty that contributes to the risk calculation can be diminished at each stage. Risk in contained field trials can be reduced at two levels-first, by careful planning and foresight during the early stages of research and development in the laboratory, and second, by thorough preparation for the conduct of the trial. Design recommendations relevant to risk reduction at the trial site are detailed under Section 7.

\footnotetext{
§Adapted in part from: Risk Assessment for Arthropod Vectors. Vector-borne and Zoonotic Diseases 2003;3:6973; and, Hoy, MA. Evaluating potential risks of transgenic arthropods for pest management programmes. In: Status and Risk Assessment of the Use of Transgenic Arthropods in Plant Protection. Austria: IAEA-TECDOC-1483; 2006: 121-144.
} 
The remainder of this section provides points to consider with regard to the genetic engineering strategy, which will increase the likelihood of accurate assessment of risk should a containment failure occur. Researchers, in collaboration with relevant oversight bodies and regulatory authorities, must think in advance about how and when to collect the information that will be necessary to make informed decisions about risk reduction and to achieve the highest possible level of confidence about their risk assessment. Tools such as mathematical modeling and computer simulation can contribute to predictive capability. However, because risk is dependent on probability, there will always be a degree of uncertainty. Uncertainty need not present a barrier to the conduct of trials, but this makes it imperative that project leaders be honest about the nature of existing uncertainty as they seek to move from the laboratory to contained field trials. Researchers must make every effort to reduce uncertainty and prevent risk. They then must work with the host country and local communities, ensuring fair process (i.e., adequate opportunities for communities to review data, express concerns, and participate in decision making), to reach agreement about the level of risk that they find mutually acceptable in the context of the potential benefits of the research.

Examples follow of specific questions to be considered for relevance in a case-by-case risk assessment for a contained field trial of GE vector mosquitoes, according to the phase of testing described in Section 5.

\subsubsection{Laboratory studies}

- Where is the genome alteration in those strategies that involve inserted genes in fixed locations in the genome? What is known about the natural function of the insertion site?

- Is the entire sequence of the DNA insertion known, and are the coding sequences defined?

- Does the genetic manipulation have any effect on longevity or other fitness characteristics of the transgenic mosquito?

- Does the product of the inserted sequence play any known natural role in the target mosquito or other nontarget species (e.g., immunity, host-seeking behavior, reproductive capacity)?

- Does the alteration have the intended effect of decreasing vector competence and transmission of the pathogen under study?

- Does the inserted sequence encode a product likely to increase the vectorial capacity or vector competence for other pathogens that it is known to transmit or that may be of special concern?

- Does the inserted gene unintentionally carry the potential to affect the ability to control the target mosquito using existing measures (e.g., affect insecticide-resistance)?

- Does the transgene product alter the immunogenic profile of the salivary gland proteins relative to wild-type mosquitoes?

- What is known from other systems about the stability of the alteration in this organism (e.g., mode of inheritance, rate of mutagenesis)?

- What types of events (random mutagenesis, rearrangement, point mutation) might result in loss of function of all or any functional part of the transgene insertion? Can the rate of such events be measured or predicted?

- Is the alteration constructed in such a way that loss of function would be disabling (e.g., the drive mechanism would not spread in the absence of the effector mechanism)?

- Is the alteration constructed in such a way that mosquitoes carrying it could be eliminated from the population (recalled) if it was not performing as intended?

- Does the inserted sequence have the capacity to mobilize? Might more than one insertion site be affected? Could insertions accumulate over time? If so, what effect might be predicted?

- Is the alteration constructed in a way that optimizes specificity for the target mosquito population and minimizes opportunity for spread to nontarget species? What is the probability of transfer of the transgene to other organisms (horizontal transfer) with which the genetically-engineered mosquito (or its remains) may come into contact?

- What methods are available to detect transgenic material for monitoring purposes? How long does the transgenic material persist after the death of the GE mosquitoes? 


\subsubsection{Pretrial testing}

- Is the target pathogen transmitted locally?

- What are the local vector species? What are the dispersal and gene flow patterns for local vectors?

- What other pathogens are transmitted locally by the target mosquito?

- Could the GE mosquitoes be eradicated by traditional methods (e.g., spraying) if there were an accidental breach of containment? Would such remediation methods have any inadvertent adverse effects, such as on beneficial insects or the environment?

\subsubsection{Contained field trial}

- Does the alteration have any unexpected effects on fitness that increase vectorial capacity, such as immature development rate and survivorship, sex-ratio of emerging adults, adult longevity, male mating competitiveness, female mating success, fecundity and fertility, intraspecific immature competitiveness of transgenic and wild type strains, host-seeking and feeding behavior?

- Does the alteration effect any phenotypic changes relevant to anticipated control methods, especially resistance to insecticides?

- If appropriate to the specific sequences inserted, is the alteration stable, as measured by copy number, sequence, and location over several generations?

- What is the relative fitness of the GE strain relative to wild-type mosquitoes (i.e., probability of persistence and spread)?

- How sensitive, specific, and reliable are detection methods for distinguishing transgenic individuals or material from wild mosquitoes?

- Can the transfer of the transgenic DNA to other organisms be effectively measured?

- How long does transgenic genetic material persist in the environment after the death of genetically-engineered mosquitoes? How long will monitoring for the persistence of transgenic material in the environment be conducted?

- If substantial persistence is observed, what measures (e.g., autoclaving, cross-linking) can be used to minimize persistence and facilitate appropriate disposal?

\subsubsection{Modeling}

- What is the predicted rate of spread of the genetic alteration into the local vector population?

- What is the relationship between the number of GE mosquitoes released and the trait frequency in the local population?

- Following the spread, what is the equilibrium frequency of the persistent transgene?

- What is the predicted effect on transmission of the subject/targeted pathogen?

- At what frequency would genetically-altered mosquitoes substantially reduce disease locally?

- How fast might a remediation or recall strategy be expected to rid the population of the new phenotype should it have undesirable effects?

\section{RECOMMENDATIONS FOR TRIAL CONDUCT: MANAGEMENT, CONTAINMENT, SURVEILLANCE, AND REMEDIATION}

As a transition phase between controlled laboratory environments and the open field, the intent of caged field trials is to gain valuable biological information in the safest way possible about the experimental strategy under study. The optimum design of a physical containment structure for caged field trials would provide for the greatest possible simulation of the natural environment while preventing movement of study organisms, as well as their genetic material, from inside the structure to the open environment. Similarly it should prevent contact between organisms outside the cage with those in it. In this section, recommendations are made on ways to minimize or abrogate the specific risks associated with potential escape of GE mosquitoes through the correct choice of materials, design, and location of contained field cage facilities. Because it is impossible to provide an absolute guarantee that no mosquito will ever escape from the caged facility, recommendations are also included for ways to minimize risk in the unlikely event of an escape through: 1) the design of a monitoring system for early detection of 
escaped mosquitoes or associated hazards, and 2) intervention strategies to avert or remediate any harm that might result should an escape occur.

\subsection{Management Practices}

The Project Plan must clearly describe the administrative structure of the project, identifying lines of decision-making authority and responsibility for all facets of the trial. This will be particularly important for international collaborative studies, where the practicality of sharing management responsibility and decision-making authority between on-site and offsite collaborators requires serious consideration. Guidance available for contained field research studies of other GE organisms ${ }^{* *}$ may provide useful insights into the range of management issues that should be anticipated in advance of the trial.

A system for record-keeping and reporting must be established to document trial activities and comply with regulatory and other requirements. Consideration should be given to maintaining a back-up set of records in a second location.

A leader of the field research team, who will be responsible for conduct of the field trial, should be designated. Depending on the structure of the program, this individual may be the principal investigator for the overall project or a designee; however, there must be absolute clarity about who is the single party with ultimate responsibility for the conduct of the trial. The field team leader should have the prerequisite scientific, administrative, and supervisory experience, including a demonstrated understanding of the requirements for operating a mosquito containment facility. This individual must play an active role in the pretrial testing of the containment facility in order to de-

**Examples include "The Recommendations for Management Practices for Field Trials with Bioengineered Plants" (http://nabc.cals.cornell.edu/pubs/Recomm_final. pdf), prepared by the National Agricultural Biotechnology Council, and "Biological and Operational Considerations for Bioconfinement (www.nap.edu/catalog. php?record_id=10880\#toc) developed by The National Academy of Sciences, USA. Legislative guidance may also be available in some jurisdictions. velop the necessary familiarity with the facility and the protocols established for its operation.

Responsibilities of the field team leader will include supervising of scientific studies, ensuring that necessary approvals have been obtained, overseeing the operation of the facility, supervising the training of personnel, ensuring compliance with all protocols and procedures to maintain containment as well as all permit requirements, and notifying the appropriate authorities of any changes that might affect risk assessment. Other key administrative positions that should be identified, with roles and responsibilities established in advance of the trial, will include the individual responsible for training in and compliance with SOPs related to security and access to the facility, and the individual responsible for maintenance and repair of the entire facility, including all security devices and barriers as well as equipment to be used for decontamination and remediation.

\subsubsection{Safety and security oversight}

The more transparent the decision-making process regarding safety and security issues can be made, the more likely the project will be to avoid the perception that a conflict of interest could result in scientific concerns taking precedence over safety. The Working Group strongly recommends that safety and security should not be compromised, and therefore further recommends that decisions on issues of safety and security involve at least one individual not directly connected to the trial or dependent on the outcome of the project. The individual addressing safety and security oversight might be the institutional safety officer, reporting to the institution with which the containment facility is affiliated and its institutional biosafety committee. ${ }^{++}$Alternatively, someone from the local nonscientific community, or an independent scientist not connected to the project, could be identified as a safety liaison to serve in this role. This individual would share authority for deci-

\footnotetext{
${ }^{++}$For example, as described in the "NIH Guidelines for Research Involving Recombinant DNA Molecules" (www4.od.nih.gov/oba/rac/guidelines_02/NIH_Guide lines_Apr_02.htm\#_Toc7261585).
} 
sions required to maintain containment, safety, and security, including those that might directly affect the conduct of the scientific studies, such as project termination. A plan must be in place prior to the initiation of the trial describing the procedures to be followed in the event that the field team leader and the independent safety officer or safety liaison disagree concerning a decision. In urgent situations (Section 7.4), this may require that the decision that is the most drastic in ensuring safety and security will be the decision taken.

Periodic internal review and adjustment, as well as periodic independent auditing, are recognized as necessary components of system safety management. The former may be accomplished by appointment of a safety oversight committee, including the key administrative staff described above and the independent safety officer/liaison, which meets frequently to share information and discuss decisions. Consideration should be given to including a member of the community (such as a liaison to the community council) on the committee and/or making committee meetings open to the public. Minutes of these meetings should be entered into the project records, and made available to requesting authorities and to the public.

It is understood that the facility will be subject to inspection by regulatory authorities. The Working Group further recommends that a plan be developed for periodic assessment of facilities and operational practices by an independent entity unaffiliated with either the project or the involved institution(s), in a manner somewhat analogous to the independent auditing of clinical trials, for adherence to SOPs.

\subsection{Physical Containment}

Physical containment can be supplemented by ecological containment (ensuring that the habitat immediately around the cage structure is not amenable to survival of transgenic mosquitoes) and biological containment (constructing transgenic mosquitoes in such a way as to limit their ability to survive or reproduce

\footnotetext{
¥¥ICH Guideline for Good Clinical Practice, Section 5.19, www.emea.europa.eu/pdfs/human/ich/013595en. pdf
}

and/or pass the transgenic material to nontarget organisms). Considerations regarding biological containment have been discussed above. Suggestions for ecological containment to enhance physical containment precautions are incorporated within the recommendations below.

Establishment of a site for contained field trials will likely require ready access to a field laboratory facility for husbandry of mosquito colonies, conduct of monitoring analyses (Section 7.3.1.1.), and other research. This laboratory should receive biosafety inspection and approval from the relevant academic or public health institution. SOPs for activities conducted in the field laboratory with wild-caught or GE mosquitoes should refer to the previously published guidance for laboratory research (e.g., Arthropod Containment Guidelines 2003). SOPs will be required for safe transport of mosquitoes between the field laboratory and field cage.

\subsubsection{Location}

Selection of the location where cages will be constructed should be based on discussions among the investigators, local communities, and the appropriate regulatory, public health, and government bodies. Criteria for selecting the location may include: ecological relevance; existing infrastructure that can be modified into a field laboratory; relative isolation from adjacent communities (but not so remote that it will complicate simultaneous field research with local mosquito populations in the case of urban vectors); relative ease of securing the area; and consent of the local communities, local authorities and appropriate regulatory/ government agencies (also see Section 8). Local mosquito populations should be studied to characterize biological traits such as population dynamics, dispersal, genetic structuring, development, and ecology. Understanding the local mosquito population will support detection of escaped insects and design and implementation of remediation should this become necessary.

Cages should be built on stable ground. Enclosures should be positioned so as to prevent 
risk of damage to the structure from falling tree limbs, flooding, fire, and other such disasters. A mosquito habitat free zone around the structure would further minimize survival and establishment of escaped arthropods. This can be accomplished through clearing of vegetation around cages and regular spraying of insecticides, if appropriate and registered for use (carefully conducted such that it does not drift toward cages). Alternatively, a "trap crop" approach, consisting of thick vegetation and artificial breeding sites placed adjacent to the cage, may be considered in some circumstances to minimize dispersal of any escaped mosquitoes. If this approach is used, the habitat should be monitored regularly and, if appropriate, treated with insecticide or an insect growth regulator to prevent mosquito production (see Section 7.3.1.). Options for monitoring escape include, but are not limited to, the approaches suggested here. Investigators will need to select the methodology that is locally most effective based on site specific data. Running a water channel around the cage may limit entry of ants and vermin, but care also must be taken that this is appropriately treated and maintained to discourage breeding of wild mosquitoes.

\subsubsection{Materials and design}

It is anticipated that cages will have mesh siding and ceiling. Commercially available greenhouses or screenhouses may be used as a basis for cage construction, although modifications likely will be required for security, adaptation to environmental conditions, or protection against natural hazards such as storms or earthquakes. Double-wall siding with 120-200 mesh/square inch $(1.2 \mathrm{~mm} \times 1.2 \mathrm{~mm})$ is appropriate for most mosquitoes; however, if exclusion of smaller mosquitoes or biting flies, such as sand flies, is required the mesh size would need to be reduced. The space between the layers of mesh can be wide enough (0.5-1.0 $\mathrm{m})$ to allow monitoring personnel to walk between layers to check the cage integrity and set traps to monitor for escaped mosquitoes. Various qualities and strengths of mesh are available. The mesh selected should be resistant to ripping, rusting, tearing, and stretching. At- tention should be paid to KLY ratings (a measure of ultraviolet radiation energy per $\mathrm{cm}^{2}$ area per year), which will provide an estimate of the life span of the mesh depending on the intensity of ultraviolet light from the sun at the field site location. The KLY value is indicative of the frequency at which material ought to be replaced due to it becoming brittle. SOPs should contain a plan for replacement of mesh before it becomes worn and in such as way that containment is not compromised. Ultraviolet coating for mesh should be used whenever possible. Rainfall should also be taken into consideration in the choice of ceiling material. Open-mesh roofs, although ideal for simulating a natural environment, may prove problematic; for example, torrential rains may damage interior oviposition habitats. Preliminary testing of materials used for enclosure construction is recommended, and may lead to alternative approaches such as overlaying or replacing the roofing mesh with a solid covering. Size (porosity) and coloration of the mesh and composition of the ceiling will affect air movement, temperature, and humidity within the cage, requiring consideration of the need for ventilation and cooling mechanisms. Care must be taken in the design of ventilation/cooling systems to ensure they do not allow mosquitoes to escape or other organisms to enter the cage.

Design of the cage foundation is another critical aspect of the construction. Better simulation of natural environment will be achieved if the floor of the cage is composed of soil with locally appropriate vegetation. Potted vegetation may enable control over the amount of growth. Soil drainage of rainwater is an important consideration, and an open bottom would facilitate more natural drainage of the ground within cages. However, risk assessment may determine that placing the cage directly on the ground does not provide the appropriate level of containment for testing of GE mosquitoes. In that case, a foundation of solid concrete overlaid with soil would provide a compromise. A raised platform may provide a useful alternative in locations where there is a chance of seasonal flooding. Foundation posts should be placed in a concrete footing in accordance with good building practice and lo- 


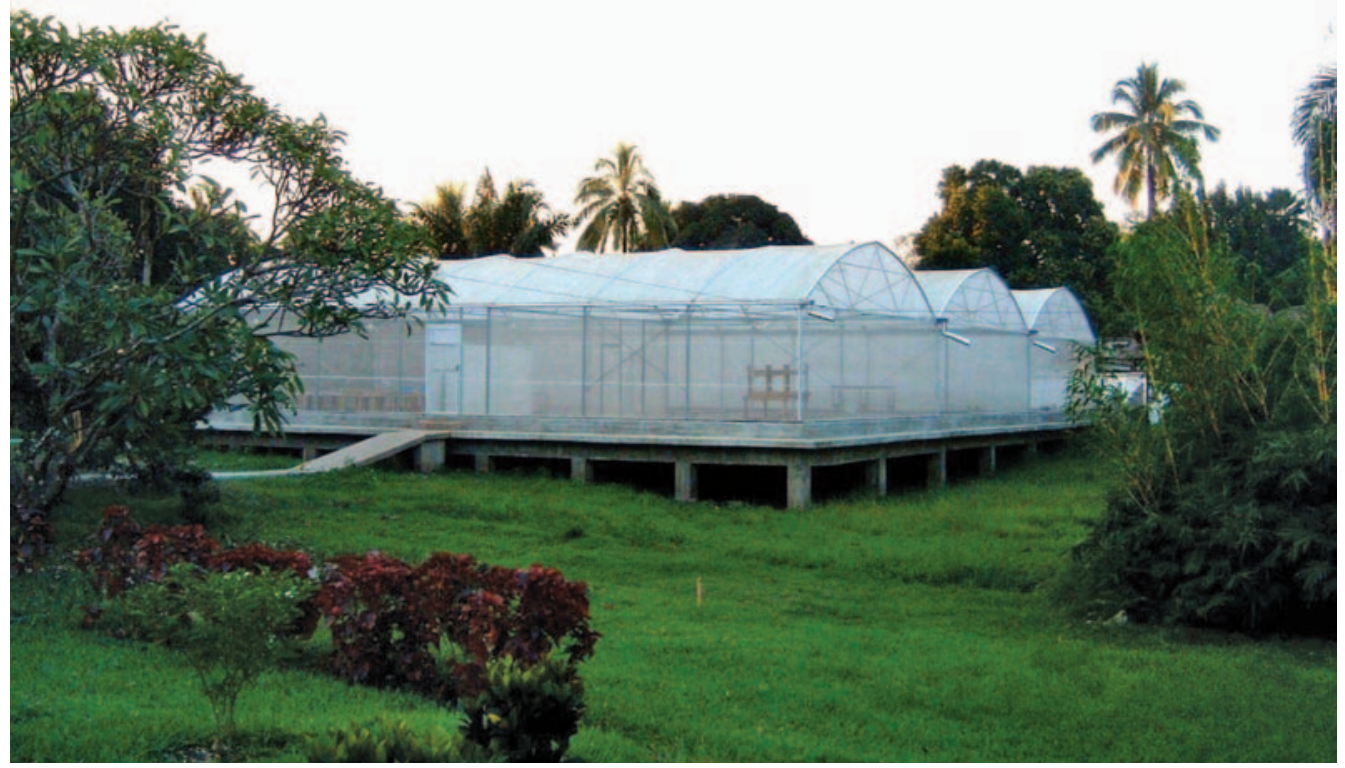

FIG. 2. Modular mosquito containment facility at the Ifakara Health Research and Development Centre in Tanzania. (Photo courtesy of Dr. Michael Gottlieb.)

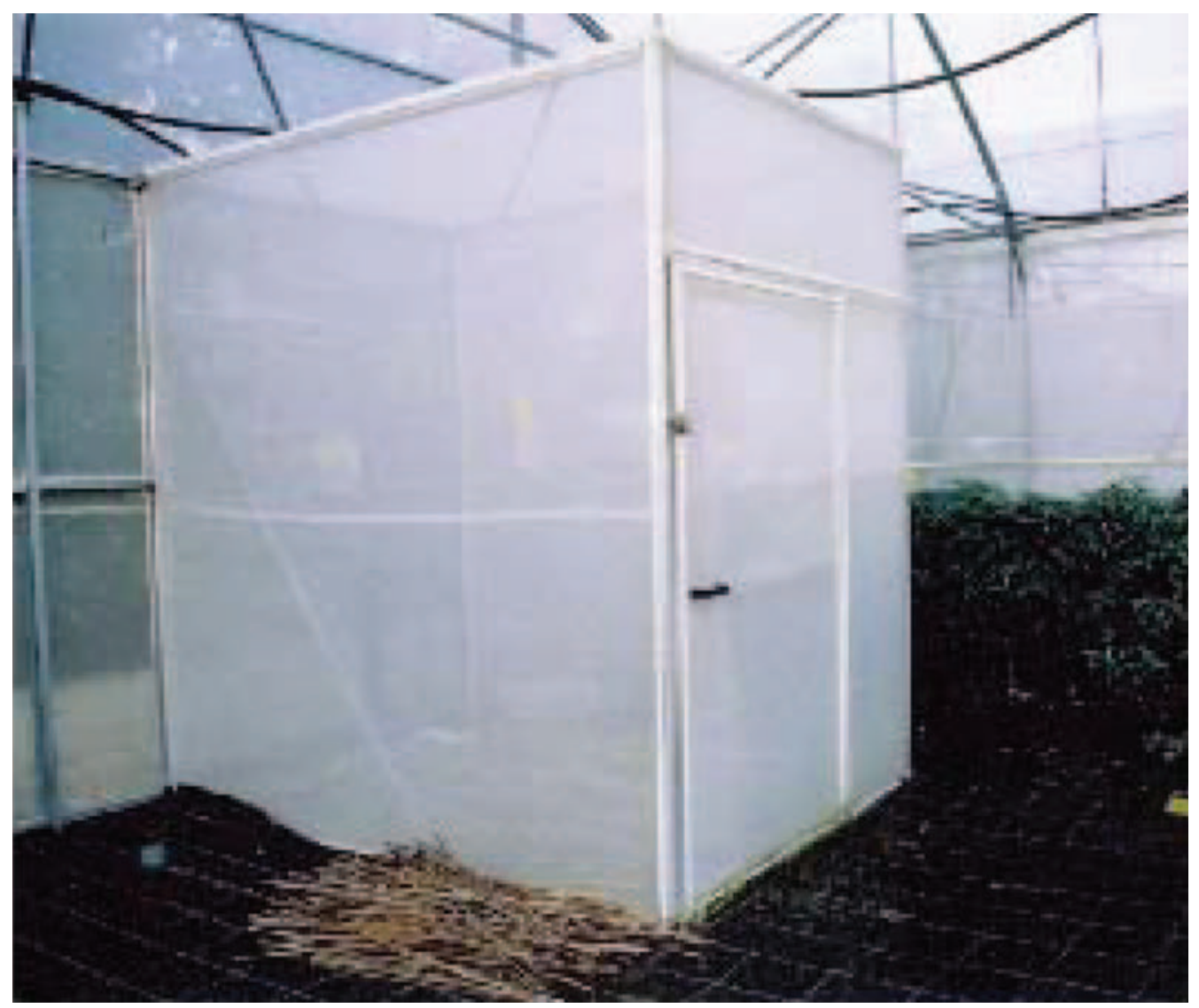

FIG. 3. A containment vestibule for entry into individual cages to enhance protection against escape. (Photo courtesy of Dr. Bart Knols.) 


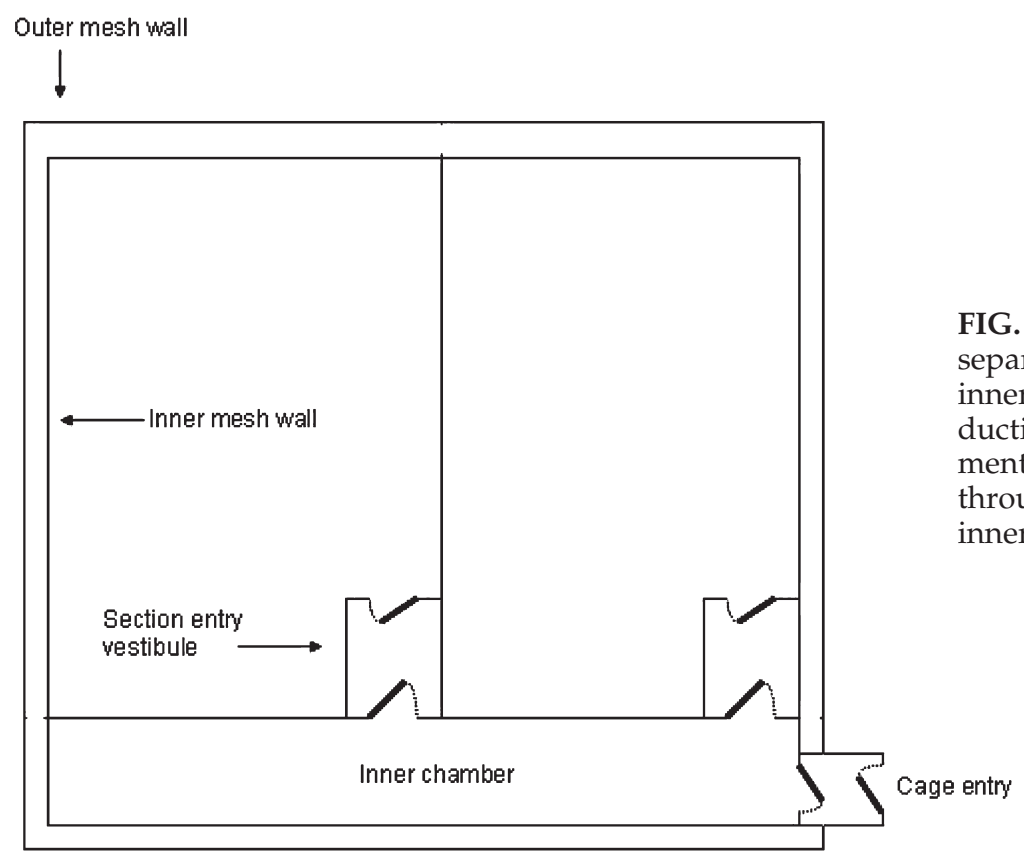

cal codes to ensure that the structure is stable. Support posts and frame should be resistant to rust, termites, etc.

Drainage and the security of cage drains is an important design feature. Although adequate drainage is required to maintain the habitat, drains provide an opportunity for the entrance of animals (arthropods, insectivorous vertebrates, etc.) as well as the exit of transgenic material into the open environment. Careful thought should be given to methods for blocking ingress and egress through drains (such as placement of water permeable barriers and plumbing traps within the drainage pipes). A raised platform design would facilitate collection of drainage water from cages for testing and decontamination treatment. Laboratory studies should determine whether simple methods for treatment of drainage water, such as boiling or chlorination, will destroy the transgenic material.

Larger units may be constructed in modular fashion using separate experimental enclosures, as in the prototype pictured in Figure 2, or specifically designed. The problem of balancing physical containment with simulation of natural habitat can, to an extent, be diminished by adding higher-order containment levels in selected parts of the field cage system, as exemplified in Figure 3. Entry and egress of personnel, equipment, or animals (Section 7.2.4.) to the facility poses a particular challenge because this is a potential opportunity for escape. A small corridor within the enclosure is recommended, because it will constitute a barrier to mosquito escape through entry points into the cage, and it will connect subenclosures in each unit. Entry into individual areas should be through a vestibule or double-screen door. An air-lock (negative air pressure chamber to facilitate capture of any escaped mosquitoes) between the doors would further strengthen containment, although this may not be suitable to all field conditions because of issues such as overheating. A minimum of four doors or levels of separation from the outside cage environment are recommended, as shown in Figure 4 . The cage must be designed to allow easy entry and exit of any animals serving as vertebrate hosts, which may require a separate entry and provision of a quarantine area for decontamination. Additional procedures and practices for preventing escape during entry and egress are discussed below.

\subsubsection{Securing containment facilities}

The level of security required will be dependent on the specific circumstances of the trial. Both risk assessment and community concerns 
must be addressed, and care should be taken to design security measures that are acceptable to and appropriate for the local community. It is recommended that the cages be protected from trespassing and pest animals by a security fence. In situations where additional precautions are needed, the fence may be lined with razor wire along the top, and page wire can be used to exclude animals. Trapping and regular monitoring for rodents and other vermin should be conducted. Partial walls or trenches around the cage can provide enhanced protection against incursion of animals. Signs should be placed appropriately around the perimeter.

Options to provide a higher degree of security include erecting a concrete wall, perhaps topped with glass/razor wire, around the entire facility and/or surrounding the perimeter of the facility with a vegetation-cleared zone equipped with motion detectors and a second security fence. If advisable, additional security may be provided by installing alarms or security cameras, and/or by employing guards to watch the facility 24 hrs per day. Background checks should be conducted on guards, as well as on other security personnel if possible.

A detailed security response plan should be in place describing responsibilities and actions to be taken in event of a breach.

\subsubsection{Operating procedures and training}

SOPs and protocols should be developed by the project team well in advance of the study. These should be described clearly and made available in the language(s) of local employees. All employees, even those not directly participating in the trial itself, should receive instruction on the goals of the study, because they provide an important bridge to the larger community. The rationale for precluding entry of other organisms into the cage and preventing the escape of mosquitoes should be thoroughly explained to all employees, and they should then be questioned to ensure understanding.

During the pretrial testing of the cage, all procedures (including the procedures for transportation and receipt of test mosquitoes) should be rehearsed prior to the introduction of mosquitoes, to demonstrate proficiency in meeting containment and security requirements. Placements of equipment, containers, etc., within the cage should take into account preventing the possibility of accidents. SOPs should provide for training on scientific, maintenance, and safety procedures for staff involved in all aspects of the trial, to be repeated regularly, and include a plan for routine oversight of employee performance. Depending on circumstances, appropriate oversight may require that at least two staff members be in attendance at all times. A mechanism should be in place for regular internal review of SOPs and protocols, and adjustment to accommodate lessons learned.

Employees will need to enter the cages to conduct activities such as egg collection, monitoring water levels in containers, caring for blood hosts, and inspecting the cage interior. Consideration should be given to the clothing to be worn for these activities: lightweight, light colored, protective clothing may be helpful both to prevent mosquito bites within the cage and to maximize the possibility of detecting any insects that might be inadvertently carried into or out of the cage. A pressurized air tank located at the door entrance is recommended as a method to decontaminate clothing before leaving the inner cage compartment. Clothing and footwear can be left inside the cage vestibule for future use, and should not leave the facility without first being freed of insects. The alternative of disposable clothing should be considered, if feasible. Additional safeguards could include the use of a shower or dunk tank upon entering and exiting the facility. SOPs should be designed to minimize the frequency of personnel entrances into the facility. SOPs should require that employees report illnesses or symptoms matching those of local mosquito-borne infections to their supervisor immediately, and before entering the cage. Entry and exit records should be maintained, including a self report of health status upon entry.

A vertebrate host for mosquito blood meals will often be essential for conducting studies in enclosures. These animals should be managed according to internationally recognized standards for the care and use of animals in research. Feeder animals should receive regular 
veterinary care and be free of mosquito-borne pathogens known to circulate locally. They should be visually inspected before entering the facility. Movement of these animals within the cage may need to be restricted to minimize the risk of damage to the structure. Wastes from animals within the cage should be appropriately decontaminated. During the exit process the animal should again be inspected to ensure it is not carrying mosquitoes.

\subsubsection{Containment considerations for Aedes and} Anopheles mosquitoes

The design and conduct of caged field trials on genetic strategies to limit disease transmission will differ according to the characteristics of the vector under study. For illustrative purposes, more detail is provided for two important vector mosquitoes-Aedes aegypti, a vector of dengue, yellow fever, and chickungunya viruses, and Anopheles spp., vectors of malaria parasites. $\S \S$

\subsubsection{Aedes aegypti}

Because Ae. aegypti mosquitoes prefer to rest indoors, mock houses built with local materials in the local style may be placed within enclosures. Appropriate resting places (clothing on hangers) and local vegetation may be placed indoors and outdoors, respectively. Water storage containers and other suitable habitats may be used to provide oviposition sites for mosquitoes. Other relevant features may be placed inside and outside of houses in order to simulate the natural environment appropriate for the target mosquito species. During pretrial testing, the number and species of blood hosts should be optimized by carefully monitoring mosquito egg production and fecundity following blood feeding on these hosts and comparing it with known fecundity of wild bloodfed mosquitoes collected in the surrounding community.

\footnotetext{
$\S \S$ These recommendations are not intended to extend to containment of mosquitoes experimentally infected with human pathogens such as dengue virus or malaria parasites (Section 2). Published Arthropod Containment Levels (Vector Borne Zoonot Dis 2003;3:75-90) provide additional guidance for studies involving pathogen-infected mosquitoes.
}

\subsubsection{Anopheles spp.}

Some Anopheles species, such as An. gambiae sensu stricto, prefer indoor environments and mock houses may be placed within cages as a resting habitat and feeding site. Appropriate vegetation and other site-specific habitat features may be simulated within the cage. Many malaria vectors lay their eggs in standing pools of water. These egg-laying habitats should be provided within the cages and should be constructed in such manner that water seepage is prevented (for instance, by using underground plastic basins). Care should be taken to ensure that swarming/mating markers are provided within the cage environment and that the height of the facility does not compromise swarming activity.

As with Ae. aegypti, blood host species should be tested carefully and optimized to reflect survival and fecundity of wild Anopheles species within the study area. Considerations for maintaining anopheline mosquitoes under contained semifield conditions have also been discussed in detail elsewhere (e.g., Knols et al. 2002).

\subsection{Monitoring and Surveillance}

Ongoing surveillance for inadvertent escape of mosquitoes from inside the enclosures into the surrounding environment is essential. All feasible efforts should be made to ensure containment, accompanied by active monitoring to facilitate detection should GE mosquitoes escape into the immediate and adjacent areas where the cage studies are being conducted. Monitoring methods should be tested using native mosquitoes from the surrounding area during the pretrial testing phase, prior to experiments involving GE mosquitoes.

\subsubsection{Monitoring of containment}

Records should be maintained of all monitoring and inspection activities. Cages should be visually examined regularly for structural damage and mesh integrity following a detailed checklist for each cage area. During the inspection process, cages should be monitored for bird nests, bats, rodents, termites, and other animals that may create openings in the cage 
through their activities. It may be useful to have those in charge of supervising these activities sign the inspection forms. If a breach is observed, it should be sealed immediately, the cause of the breach and potential for release should be assessed, and SOPs for intervention/remediation (Section 7.3) initiated. Depending on the cage design and nature of the breach, it may be possible to close the affected section for repair without necessitating depopulation of the entire facility. Should escape of GE mosquitoes be detected in the absence of a physical breach of the facility, the most likely cause would be a breach of protocol. SOPs in this case might involve expanded monitoring, remediation in the area around the facility, imposition of tightly controlled access to the cage, immediate initiation of retraining for all employees, and reevaluation/modification of SOPs.

The focus of the monitoring activities should be tailored to the behavioral characteristics of the vector species being studied. For example, Ae. aegypti is particularly amenable to collection in and around homes, because it tends to be found with humans and readily lays eggs in artificial containers holding small amounts of water. Numerous studies in a variety of locations indicate that most males and females in this species do not move more than $100 \mathrm{~m}$, based on 14 days or more of daily recaptures (Harrington et al. 2005). However, humanaided movement of adults, larvae, pupae, and eggs in containers may be much farther, and should be considered a mechanism for rapid spread of novel genetic material. Monitoring systems should take this possibility into account. Greater dispersal ranges (up to $1 \mathrm{~km}$ per day) have been reported for adult An. gambiae and other Anopheles spp.

In the case of Ae. aegypti, traps should be present continuously within the entry vestibules of the cage and each section (Fig. 4), internal cage corridors, and immediately outside structures. For container-breeding mosquitoes such as Ae. aegypti, placement near the cage of vegetated "trap crop" areas that are populated with large flooded containers such as tires or buckets, to encourage localized oviposition by any escaping mosquitoes, could be considered. If used, these containers should be treated with an insect growth regulator to prevent production of adult mosquitoes while maintaining larvae that can be sampled and tested for presence of transgenes. Other types of traps, such as bednet, window, and animal-baited traps, may be more appropriate for Anopheles vectors. The best approach for monitoring containment is not limited to the options mentioned, and will need to be selected according to local effectiveness based on site-specific data.

Inspection of traps should be conducted daily. Thorough monitoring of the environment surrounding the cages should be conducted at least once weekly while studies are in progress. For Ae. aegypti, this can be done through aspiration collections and distribution of standard ovitraps, BG or similar traps. Aspiration collections can be made from black resting structures containing an oviposition container deliberately constructed at an appropriate radius outside of the cage where adult Ae. aegypti are likely to rest. Ovitraps and other potential breeding sites should be checked at a frequency sufficient to preclude the emergence of adult mosquitoes. Ovitraps should be placed around cages at an appropriate radius around the field station. Diligent effort should be made to remove or cover all potential development sites in the survey area and to minimize competition from natural larval development sites. Monitoring systems may be set up in communities or other potentially receptive habitats adjacent to the field site. Adult mosquitoes may be sampled systematically by aspiration from inside homes or with species appropriate traps. Immature forms may be sampled from their aquatic habitats and/or with ovitraps.

For Anopheles vectors, animal-baited traps or other appropriate traps should be placed at an appropriate radius around the field station and operated overnight. Indoor resting collections also may be employed.

SOPs should be in place with precise details of the response, including who must be notified and when, if evidence suggests a potential release of a transgenic mosquito outside the cage. Monitoring should be intensified and the protocol should be modified as appropriate for the vector species and risk level (see Section 6.3). Immediate corrective action should be initiated to identify and rectify the cause of the es- 
cape. The response should include immediate inspection of cages for problem areas and may require prompt remediation (see Section 7.4). Records should be maintained of all actions in response to a suspected release.

\subsubsection{Assay for transgene detection}

DNA samples from pooled mosquitoes may be tested for presence of the transgene by gene amplification (polymerase chain reaction or PCR). Eggs in appropriately labeled ovitrap samples may be hatched, and first instar larvae tested. For Anophelines, eggs skimmed from water surfaces and held until hatch or first instar larvae collected from water sources may be tested. Gene amplification procedures (PCR or Inverse PCR) should be developed to monitor the inserted genes. All positive PCR reactions should be rerun for confirmation and should include appropriate controls.

The sensitivity of a PCR screen should be determined in preliminary laboratory studies to ensure that the transgene can be detected at low frequencies. Specifically, the sensitivity of the assay can be determined by combining different ratios of transgenic/nontransgenic mosquitoes. If the pooled DNA screen proves inadequate, DNA extractions can be carried out and PCR screens performed on a subset of individuals within a sample. In this case, it will be important to predetermine a minimum sampling ratio that takes into consideration variance within the sample predetermined from laboratory studies.

\subsubsection{Monitoring effects on target mosquitoes}

Studies to gather data on the behavior and genetic make-up of conspecific organisms within mixed populations in the cage environment are critical to the contained field trials. These experiments may include assessments of population reduction, population replacement, and the nature of the genetic construct after movement through several generations of mosquitoes within field cages. Routine monitoring procedures, to be conducted prior to initiation of cage studies and regular intervals thereafter, of all mosquito populations (transgenic, wildtype, and mixed) for susceptibility to insecti- cides, particularly those expected to be used in depopulation or remediation activities, should be specified in the SOPs.

\subsubsection{Monitoring epidemiology}

Procedures should be established to detect changes in the frequency or severity of the disease that is targeted by the genetic strategy under study, as well as any other vector-borne diseases whose transmission could inadvertently be affected by alterations in the target mosquitoes or other local arthropods, in the vicinity of the field cages. Routine surveillance activities should involve collaboration with local health authorities and/or clinics that can track disease observations (passive surveillance), and should be conducted regularly during the course of the study and for a reasonable period of time after conclusion of the study. Interpretation of the results of such surveys will need to take into account the inherent variability of disease incidence in endemic regions. Establishment of a "control site" remote from the cages for comparison of incidence might be considered in this regard, as this could serve to clarify whether any increase in disease reporting is related to the contained field trial. A move to active surveillance may become important if an inexplicable increase in disease is observed in the environs of the field cage or in the event an inadvertent release of GE mosquitoes is detected.

\subsubsection{Monitoring effects on nontarget organisms}

The level to which monitoring efforts must be undertaken with regard to nontarget organisms during contained field trials will be clarified during risk assessment and in discussion with project consultants (Section 5.1.1.). PCRbased testing can be employed to assess movement of the transgene to nontarget organisms within the environment. This approach can begin in the laboratory by categorizing the diversity of organisms that might be impacted at the potential field site. Appropriate monitoring activities should be conducted through the course of the studies.

A tiered approach to ecological risk management similar to that recommended for transgenic crops may be relevant (Hill and 
Sendashonga 2003, Environmental Protection Agency 1998).

Consideration of nontarget organisms to be monitored routinely must take into account cage design and location. Although it is clearly not feasible to monitor routinely all organisms in the immediate environment, a realistic plan might identify a subset of "sentinel" organisms based on their potential for interaction with transgenic mosquitoes in the field cage as well as on the findings from earlier laboratory studies. Although the cage is designed to exclude such organisms, they will be assessed if found within the facility. Such a subset might be chosen from:

- Closely-related mosquito species

- Soil microbes (bacteria and viruses), arthropods, and other organisms such as earthworms in the interior soil habitat

- Aquatic organisms living within the mosquito larval habitat inside the field cage

- Other arthropods, such as smaller flies, that may gain entry through the mesh or doorways, or reproduce within the cage

- Predatory invertebrates (such as spiders and ants) or predatory vertebrates (such as lizards).

\subsection{INTERVENTION AND REMEDIATION STRATEGIES}

SOPs must be in place in advance of the study, describing in detail the actions to be undertaken in event of a pending or proven breach of containment. The SOPs must be available in the language(s) of the local employees, and regular testing or simulations should be conducted to ensure that all employees are familiar with the criteria for decision-making and the procedures that must be implemented. It is essential that all employees know who is responsible for decision-making in this situation and that they are able to contact the responsible individuals without delay (Section 7.1.). Local public health and vector control authorities, as well as community leaders, should be consulted in the development of these SOPs, and the plans should be shared with those living in the surrounding area as part of the community engagement process. Insecticides proposed for use in remediation ac- tivities must be approved for use within the country and maintained on site, and operators should be trained in their safe use and handling. SOPs should specify routine testing of application equipment, and of the susceptibility of GE and local wild-type mosquitoes. Consideration should be given to including rapid interior depopulation capability in the design of the field cage. A communication plan should be in place for immediate use in the event of inadvertent release (Section 9).

\subsubsection{Prevention of inadvertent release}

At some sites, natural events (e.g., hurricanes, cyclones, tsunamis) could threaten the containment facilities. Daily activities at the field site should include monitoring local weather and other environmental conditions. If a natural event threatens the structural integrity of the enclosures, procedures must be implemented to depopulate all field cages to prevent release of transgenic mosquitoes. SOPs should provide detailed instruction for when to begin implementation (e.g., the level of threat that will trigger depopulation), the extent of depopulation (e.g., field cages only or indoor laboratory cages as well), and how the depopulation will be achieved (e.g., aspiration or pesticide application). Depopulation procedures should take into account the possible need to repopulate the facilities after the threat has passed (i.e., when to use and when to avoid the use of residual pesticide).

\subsubsection{Remediation of inadvertent release}

A vigorous response must be triggered if transgenic mosquitoes, or their genetic material, are found in the open environment as a result of routine monitoring. SOPs should define the degree of response as it relates to the level of risk (see Section 6.3.). Commensurate with the risk posed by the genetic strategy under study or the extent of the release, this plan should include the option of diverting project resources to implement broad-scale insecticide applications and other suitable mosquito control practices in conjunction with the local vector control personnel within an appropriate area around the containment facility. Treatment of choice will depend on the specific site 
and mosquito species or strain housed in enclosures, but likely would involve spraying inside every house with an insecticide (e.g., deltamethrin/resemethrin, malathion) and treating water containers (for Aedes) or natural breeding sites (for Anopheles) with a larvicide (e.g., temephos, Bti), and, if appropriate, biological control or environmental modification. Routine monitoring for insecticide susceptibility will provide a basis for selecting active ingredients. The scope of remediation efforts may be guided to some degree by computer simulation studies. To determine the actual extent of release and/or effectiveness of local remediation, monitoring using the techniques described above (Section 7.3.1.), should be carried out in adjacent communities, cities, and ports of exit from the study area. These efforts should be continued until transgenic mosquitoes have not been detected for a reasonable period of time following the incident. Assessment of the possible collateral environmental and ecological impact of a remediation event on the area surrounding the containment facility also should be considered, as this poses a hypothetical hazard.

\subsubsection{Remediation of adverse effects on nontarget organisms}

The method and extent of remediation will depend on the level of risk for adverse effects on nontarget organisms as a result of escaped transgenic mosquitoes or transgenic material. The extent to which such efforts would be necessary or desirable will be influenced by the specific nature of the genetic construct under study, as well as the field-site location, and should be discussed with and agreed upon by the local community. SOPs should describe the options for broad-range remediation, including specific plans for depopulation of all affected organisms within and in the immediate vicinity of the cages, as well as specific methods for monitoring the effectiveness of remediation actions.

If possible, efforts should be made to understand the nature of the nontarget effect. This may involve returning to laboratory studies for the purpose of developing another safer, more stable modification of the target organism.

\subsubsection{Remediation of effects on the target organism}

It is anticipated that adverse effects on the target mosquito species would be recognized in earlier phases of testing, either in the laboratory or during the pretrial transgenic biosafety testing, and thus it should be unlikely that the first evidence would appear as a result of an escape. Should adverse effects on local conspecific mosquitoes first be noted after escape of GE mosquitoes, however, remediation efforts would proceed as described for inadvertent release (Section 7.3.2.). A return to laboratory studies in order to understand the nature of the effect would inform the development of alternate approaches to modification before returning to field cage trials.

\subsubsection{Termination of study}

At the completion of the study, all cages should be depopulated of all life stages, using insecticides if necessary, and the site should be decontaminated to the extent appropriate and feasible for the particular system under evaluation (e.g., by autoclaving materials, chemical treatment). Leaving cages vacant for several months after depopulation may ensure mortality of any transgenic mosquitoes not reached by insecticide based depopulation. SOPs should be developed for appropriate disposal of all GE mosquito material.

\section{REGULATION OF GENETICALLY ENGINEERED (GE) MOSQUITOES}

Regulation of field trials of GE mosquitoes may occur at the following levels:

- International covenants and protocols

- National or federal

- Regional, state, provincial, or tribal

- County or local municipal

- Institution-specific requirements imposed by the organization(s) supporting or conducting the research.

At each level, the scope of relevant regulations includes not only those that explicitly refer to 
GE organisms, but also those that apply to release of any organism to the environment as well as to other trial implementation activities, such as general biosafety and research ethics/human subjects requirements, environmental health and occupational safety regulations, transportation regulations, quarantine regulations, etc.

Requirements at the location of the field trial and at the location where the pretrial laboratory research is conducted (if different) must be determined. The source from which funds supporting the research are obtained also may have significant implications for the regulatory requirements. For example, activities undertaken outside the United States but supported by funding from U.S. federal agencies are subject to certain requirements under the National Environmental Policy Act.***

There is no established precedent for government agency, international organization, covenant, or treaty regulation of GE mosquitoes specifically, other than public human and animal health import permit and quarantine regulations intended to prevent the incursion of new pests and diseases into countries where they are not indigenous. GE mosquitoes may present new challenges to agencies with responsibilities to regulate their importation, distribution, contained testing, and release or use in the environment; however, there are precedents for the environmental release of both GE organisms and exotic invertebrate biological control agents on which agencies can draw. As of this writing, the only regulatory precedent for field use of transgenic insects is in the United States. It is based on a combination of import permit procedures, a transparent documentation process, and a law that mandated preparation and public comment on two Environmental Assessments and an Environmental Impact Statement in preparation conducted under the National Environmental Policy Act. ${ }^{++}$ Agencies or authorities charged with regulation of GE mosquitoes likely will not have es-

***Executive Order 12114, Environmental effects abroad of major Federal actions, 44 FR 1957, 3 CFR, 1979 Comp., p. 356.

${ }^{++}$Examples of Environmental Assessments for transgenic pink bollworm (a Lepidopteran pest of cotton) may be found at www.aphis.usda.gov/biotechnology/ea/ pdf/aphisdocs/05_09801r_ea.pdf tablished processes or personnel to regulate them specifically. For early trials involving GE mosquitoes, they may be inclined therefore to rely on experience with conventional organisms that are typically quarantined or transgenic plants.

\subsection{Regulatory Costs}

Authorities at the different levels of regulation should understand that funding resources for research, development, and regulatory risk assessment data requirements for insect vectors of tropical diseases are likely to be considerably less than for commercial transgenic crops. Lack of commercial interest in GE mosquito technology, because of the lack of profit incentives, will limit resources available to pay for development, risk assessment data generation, registrations, permits, long-term monitoring, etc. This limitation may be offset at least in part by the ability to provide greater transparency in the GE mosquito risk assessment process than might be the case for a commercial product (where corporate confidential business information may be involved), which could help to facilitate regulatory approvals and public acceptance for introduction, contained caged field-testing, and eventual release in the environment.

In consideration of the costs that may arise in the development of risk assessment information and data, regulatory agencies must be encouraged and assisted to define scientifically based requirements. Additionally, the agencies should consider how they might participate in the studies by providing grants or other institutional contributions, such as labor and collaborative agreements for safety studies.

\subsection{Regulatory IMPACT}

Regulatory issues may arise in at least two phases of a contained field trial. First, early in the process of selecting the field site for the trial, it is important to identify all applicable regulations to the field trial and to understand how they may affect the research. Ethical, social, and cultural considerations for selecting a field site, including several regulatory and administrative issues, have been discussed in detail elsewhere (Lavery, Harrington, and Scott, manuscript submitted). Briefly, questions to be 
considered during the site selection process include:

- Are there conditions that might encourage interest in the application of alternative solutions to disease control (e.g., increase in disease incidence, rise in vector population or introduction of a more effective vector, emergence of resistance to current control methods in the pathogen or vector, difficulties in obtaining or sustaining other control options)?

- Is the government generally supportive of new biotechnologies?

- Does the country subscribe to any international agreements related to use of GE organisms? If so, what are the mechanisms and who are the government officials responsible for complying with these agreements?

- Are there existing laws or regulations related to GE organisms? If so:

- Do the regulations specifically apply to cage-contained research?

- Do the regulations specifically apply to blood feeding insect vectors of human disease?

- Is there a requirement for risk assessment information?

-What are the information requirements?

- Is there any effort underway to add new or change existing legislation that might affect the upcoming trial?

- Are there other relevant regulations for oversight of research (e.g., biosafety, protection of human subjects) and mechanisms for meeting these requirements (e.g., duly constituted institutional committees)?

Second, after a site has been selected and the relevant regulatory requirements identified, the regulations likely will impose obligations with respect to monitoring and reporting the trial implementation and outcomes. Therefore, arrangements must be made to comply with these and all other permit requirements.

\subsection{INTERNATIONAL ORGANIZATION AND COVENANTS}

The emphasis of nearly all international bodies concerned with GE organisms is predomi- nantly with food crops and their products, which are imported and used for direct human or livestock consumption, food or feed processing, or for planting and propagation. GE animals and microorganisms for fermentation, pharmaceuticals, vaccines, industrial chemical, and livestock production improvement have also been traded internationally. Presently, because of the lack of historic demand, there is little international regulatory emphasis on GE insects. The North American Plant Protection Organization has developed guidelines for importation and contained field release of transgenic arthropods that are plant pests (www. nappo.org/Standards / Consultation / R S P M 27 / R S P M \% 2027 - V e r s i o n March\%205\%202007-e.pdf). The International Atomic Energy Agency (IAEA) and United Nations Food and Agriculture Organization (FAO) have produced a publication addressing risk assessment for transgenic arthropods in plant protection (www.pub.iaea.org/MTCD/ publications/PDF/te_1483_web.pdf). An international effort has been undertaken to establish guidance for the regulation of new biotechnologies related to crop pests and human disease vectors (http://biopesticide.ucr.edu/ daegu/daegu.html).

Although many of the international covenants place high importance on public health, to the extent public health transcends the terms of the agreements, the most directly applicable international covenant is the Cartagena Protocol on Biosafety. However, only a few articles in it apply to caged field testing of GE mosquitoes. In addition, other international agreements dealing with GE organisms (see Sidebar on pg. 32), including those regarding trade, have preemptive emphasis on human health; these may have more relevance to the possible eventual distribution and implementation of GE mosquitoes for control of vector-borne diseases than to the current discussion of contained field trials.

The Cartagena Protocol on Biosafety to the International Convention on Biological Diversity (www.biodiv.org/biosafety/default.aspx) is an international agreement on living modified organisms (LMOs). The Protocol was negotiated in 2000, and it became effective when the number of signatory joining countries reached 50; it currently includes more than 135 nations, 
including many developing countries. The Protocol affirms the precautionary approach contained in Principle 15 of the Rio Declaration on Environment and Development. One of the outcomes of the Cartagena Protocol is the establishment of the Biosafety Clearing-House for the compilation and exchange of information on movement and release of genetically modified organisms. This comprises a database that contains records for all types of national legislation, and some governments have also provided information on their biosafety regulatory framework, including summaries of regulatory frameworks, National Biosafety Frameworks, and regulatory developments (http://bch.biodiv.org/ laws/about.shtml). Whereas these national regulations take precedence, aspects of the Protocol to be kept in mind for planning of field trials of GE mosquitoes are:

- Article 4 - Scope: The Protocol applies to the transboundary movement, transit, handling, and use of all LMOs that may have adverse effects on the conservation and sustainable use of biological diversity, taking also into account risks to human health. Under the protocol, a country that wants to export LMOs for intentional introduction into the environment, must seek advance informed agreement from the importing recipient country before the first shipment takes place. The protocol provides for decisions to be based on risk assessment. Importers can ask the exporter to do the risk assessment.

- Article 6 - Transit and Contained Use: The provisions of this Protocol with respect to the advance informed agreement procedure shall not apply to LMOs in transit. The provisions of this Protocol with respect to the advance informed agreement procedure shall not apply to the transboundary movement of LMOs destined for contained use undertaken in accordance with the standards of the Party of import. Contained use means any operation, undertaken within a facility, installation, or other physical structure, which involves LMOs that are controlled by specific measures that effectively limit their contact with, and their impact on, the external environment.

\subsection{Addressing Regulatory Requirements}

The following steps are recommended to address regulatory requirements and comply with accepted standards of conduct in any specific jurisdiction:

1. Identify all applicable laws, regulations, and the regulatory officials

2. Consult with the responsible regulatory officials

3. Prepare to meet regulatory requirements

4. Prepare to meet monitoring and reporting requirements

5. Ensure a mechanism for oversight of the trial for biosafety and other compliance requirements.

For field trials on new human disease control strategies using GE mosquitoes, it would be desirable to work in a country where there is a lead agency with clear regulatory authority and responsibility willing to coordinate with other agencies or departments having less clear regulatory responsibility. However, it is possible that an established and efficient regulatory process may not be present. In some countries, regulations to govern and monitor research on GE organisms have not been instituted. In such cases, the developer and importer of the technology may have to assume a more proactive role in developing, promoting, and effectively communicating a regulatory strategy, including appropriate risk assessment criteria, to country and local authorities and possibly the public in the field test area(s). The preparation of an Environmental Impact Assessment (EIA) is a widely established practice with good likelihood of acceptance in a country where there may be limited regulatory capacity (http://biopesticide. ucr.edu/dacgu/dacgu.html) Collaboration with country regulators and scientists on the development of an EIA will also provide valuable capacity building relevant to other areas of biotechnology. This strategy is consonant with recommendations for the development of a coevolutionary regulatory approach, where safety management goes hand-in-hand with the development of the technology (Juma and Serageldin 2007).

Before committing to a field site or location, it should be determined whether there is local support for the trial in the host country from 


\section{InTERNATIONAL Organizations OR COVEnANTS with Potential Relevance to GE Vector Mosquitoes}

In addition to the Cartegena Protocol on Biosafety, several other agreements deal with GE organisms (also called GMOs and LMOs) and have emphasis on human health, including:

- Codex Alimentarius, Food and Agriculture Organization and

World Health Organization (www.codexalimentarius.net/web/index_en.jsp)

The Codex Alimentarius Commission was created in 1963 by FAO and WHO to develop food standards and guidelines under the Joint FAO/WHO Food Standards Programme. WHO has also been addressing a wide range of issues in the field of biotechnology and human health, including safety evaluation of vaccines produced through biotechnology, human cloning, and gene therapy. The Codex task force on Foods Derived from Biotechnology reached agreement in 2002 on a final draft of "Principles for the risk analysis of foods derived from biotechnology," which provides the framework for evaluating the safety and nutritional aspects of GM foods.

- International Plant Protection Convention (IPPC) (www.ippc.int/IPP/En/default.jsp)

The International Plant Protection Convention is an international treaty relating to plant health, deposited with the Director-General of the FAO and administered through the IPPC Secretariat located in FAO's Plant Protection Service. The Convention makes provision for the application of measures by governments to protect their plant resources from harmful pests, which may be introduced through international trade. IPPC has formed a working group on phytosanitary aspects of GMOs, biosafety, and invasive species, which will develop standards for environmental hazard risk analysis.

- IPPC International Standards for Phytosanitary Measures: ISPM No. 11 (2004)

Pest risk analysis for quarantine pests including analysis of environmental risks and LMOs:

The standard provides details for the conduct of pest risk analysis to determine if pests are quarantine pests. It describes the processes to be used for risk assessment as well as the selection of risk management options. Some LMOs may present a phytosanitary risk and therefore warrant a pest risk analysis.

- The Office International des Epizooties (OIE) (www.oie.int/eng/en_index.htm)

OIE is the world organization for animal health. It has three main missions: to inform members of the occurrence and course of animal diseases throughout the world and of means of controlling these diseases; to coordinate international research devoted to the surveillance and control of animal diseases; and to promote the harmonization of health regulations for trade in animals and animal products among members. The OIE has had a working group on biotechnology since 1996.

The OIE is principally concerned with animal or livestock health issues that may be associated with GM animals and vaccines.

\section{- World Trade Organization}

The WTO deals with trade issues related to marketing of GMOs (www.wto.org/english/tratop_e/sps_e/sps_ agreement_cbt_e/c8s1p1_e.htm\#gmo). The Agreement on the Application of Sanitary and Phytosanitary (SPS) Measures applies to GMOs in respect to: protection of human or animal life from risks arising from additives, contaminants, toxins or disease-causing organisms in their food, beverages, and feedstuffs; protection of human life from plant- or animal-carried diseases (zoonoses); protection of animal or plant life from pests, diseases, or disease-causing organisms; and protection of a country from damage caused by the entry, establishment, or spread of pests. Regulations on GMOs would have to conform to the provisions of the SPS Agreement, such as scientific risk assessment and least trade restrictive measures. Other relevant WTO agreements include The Agreement on Trade-Related Aspects of Intellectual Property Rights (TRIPS), which could be invoked in a dispute on intellectual property protection related to GMOs.

The 2002 WTO Agreements and Public Health joint study by WHO and the WTO Secretariat (www.wto.org/ English/news_e/pres02_e/pr310_e.htm) explains how WTO Agreements relate to different aspects of health policies. The study covers several areas including infectious disease control, environment, and biotechnology. The study explains countries' rights to take measures to restrict imports or exports of products when necessary to protect the health of humans, animals, or plants. The study discusses application of biotechnology to foods and potential heath effects such as gene transfer from plants to microbial or mammalian cells, transfer of antibiotic resistance, and allergenic effects. 
national scientists and government regulatory authorities. In the absence of national and local support, it will be difficult, if not impossible, to justify field-testing. Consultation with country research scientists, the public health community, regulatory officials, and other stakeholders is necessary to determine the degree of support to expect. A written and mutually signed collaborative project proposal with host country authorities will be useful to define expectations, delineate research activities, and establish accountability for the field trial.

\subsection{A Proactive Approach to Regulatory Approval}

If there is an established or defined regulatory and risk assessment procedure, and a respective agency or authority in the country where field testing of GE mosquitoes is proposed, the local authorities and processes will determine the type and format of any review that will be necessary to obtain regulatory approval. In the case of contained field trials, well-constructed and maintained cages may be considered sufficient containment to mitigate potential risks, which may reduce or eliminate the need for further in-depth risk assessment. However, if regulatory procedures do not exist or are perfunctory, a proactive approach by the research organization to prepare risk assessment and risk management documentation according to internationally accepted standards can make the decision to proceed with the proposed field research a better-informed decision, and can assist the regulatory and public approval processes.

\subsubsection{The Environmental Impact Assessment}

The Environmental Impact Assessment (EIA) is a widely used format to meet country government regulatory agency requirements and to provide for public transparency of the government decision process. The EIA is a document that is developed openly to the public with available scientific, societal, and stakeholder input, and the public is provided the opportunity to be informed and comment on decisions that may affect the environment before a proposed action occurs.
After researchers have informed the country regulatory agencies of proposed plans and actions, meetings and discussions should focus on coordination of the EIA process and identification of risk assessment information and data needs that are scientifically relevant and can be obtained within reasonable time, effort, and expense. It will be essential to reach an understanding on clear requirements for data necessary to support science-based risk assessment. Preliminary investigations for the EIA should involve all interested parties, such as the proponent of the field testing proposal, appropriate authorities and agencies, and appropriate members of the public, such as university or medical community professionals. The results should help determine the scope, depth, risk assessment concerns, and terms of reference to be addressed within the EIA document. In some countries, this involves public meetings, which are advertised in advance of their occurrence. The World Bank's Environmental Assessment Source Book contains a chapter on public consultation that may help to inform this process. investigative process that a decision may be scientifically justified so that a full EIA is unnecessary because of factors such as indigenous presence, commonality of the technology to the environment, substantial similarity to an existing technology or previous trial, or self-mitigation factors built into the GE mosquito construct to the extent that no environmental impacts are expected or are likely to occur.

The full EIA contains a comprehensive risk assessment and, as described in Section 6.3., is case-by-case specific to the particular GE strategy and its unique characteristics. It includes assessment of potential human effects, other animal and nontarget organism effects, effects and persistence in the environment, societal impacts, and other possible environmental impacts or effects, such as threatened or endangered species that may be affected. Appropriate alternatives (both those currently

\footnotetext{
$\ddagger \ddagger \ddagger$ Public consultation in the EA process: a strategic approach, http://siteresources.worldbank.org/INTSAFEPOL/ 1142947-1118039086869/20526299/Update26Public ConsultationInTheEAProcess AStrategic Approach May1999.pdf
} 
available and those under development) also should be equitably considered along with their potential environmental and societal impacts, so that comparisons may be easily drawn. The body of the EIA should be written in the most common language of the county for mass-communication and should be sufficiently publicized to provide opportunity for public comments.

The full EIA may lead to a finding that the proposed study will have no significant impact, and a decision will be made to proceed. Alternatively, it may lead to a decision not to proceed or to a lengthier or more rigorous assessment when a finding of no significant impact cannot be reached and more information and investigation is considered necessary.

The research organization originating the GE mosquito technology should bear much of the burden to develop the EIA documentation, because the originators are most familiar with the technology and its potential benefits, effects, and risks. If the originating organization is not located in the country hosting the trial, the EIA documentation should be developed in collaboration with cooperating scientists and regulatory agencies within the proposed country of use.

\subsubsection{EIA format}

The specific form and content of the EIA should be planned to meet the needs of the regulatory authorities of the country involved. If a format has not been developed by the country in which a trial is to be carried out, a working format based on those currently in use elsewhere might be proposed to country regulatory agencies and adapted to their specific needs. $\$ \$ \$$ Following consensus on an EIA gen-

\&\&\&Formats and other information regarding similar environmental documentation are provided in the following websites: USA NEPA format (www.nepa.gov/ nepa/regs/ceq/toc_ceq.htm), Canadian Environmental Assessment agency (www.acee-ceaa.gc.ca/), Euro-pean Union (http://ec.europa.eu/environment/eia/home.htm; http://ec.europa.eu/environment/biotechnology / index_en.htm; (www.europa.eu.int/comm/environment/ eia/eia-guidelines/g-review-full-text.pdf), Australian Government Department of Environment and Water Resources (www.environment.gov.au/epbc/about/index. html; www.deh.gov.au/epbc/assessmentsapprovals / flowchart.html), Convention on Environmental Impact Assessment in a Transboundary Context (Espoo 1991) (www.unece.org/env/eia/eia_text.htm) eral content format, the format may be proposed for adoption by regulatory agencies as a publicly-transparent tool for risk assessment and regulatory approval of field-testing of GE mosquitoes.

\section{COMMUNITY AND PUBLIC ENGAGEMENT}

Successful implementation of caged field trials of transgenic or biologically modified insect vectors will depend not only on meeting formal regulatory requirements, but also on gaining and maintaining acceptance within those communities selected for the trials, and within the broader society of which these communities are a part. Failure to gain this acceptance can lead-and in the past has led-to early termination of projects before completion.

\subsection{A BASIC MOdEL \\ of Community ENGAgEMENT}

Several of the terms used to discuss these issues-none more so than "community" - have imprecise and often multiple connotations. For the purpose of these recommendations, the term "community" is used to refer to those people who consider themselves to belong to the area directly affected by a planned trial, and to the informal and formal institutions linking those people with one another, such as village or town councils or voluntary organizations. "Community," in this sense, is a nested concept. In any given setting, there are likely to be communities within communities; that is, within the broadly defined community, there will be clusters of people who share additional ties, especially through shared residence in a particular locality but perhaps also through shared values, shared history, etc.

Community engagement refers to activities and processes undertaken by or on behalf of the researchers and involving residents and other representatives of communities affected by a planned caged field trial, with a view to negotiating a mutually acceptable set of terms and conditions for proceeding with the trial. In the context of field trials, the possibility that authentic communities might be nested within one another may require multiple community engagement strategies and negotiations. 
Although numerous accounts of community engagement have been published, the literature does not reveal any single, empirically tested, and universally accepted model of community engagement. The basic model described here represents a distillation of published models, focusing in particular on common elements. Although some of these steps are preconditions for others, the steps should not be seen as a linear sequence. Rather, work on most of the steps should proceed simultaneously.

\subsubsection{Define and explain the nature and purpose of the trial}

It is important to make available at the outset a clear, nontechnical description of the trial, the thinking behind it, its objectives-both short and long term-and the activities involved in carrying it out. This description should be available in the local language(s) and communicated in ways that are genuinely accessible to community members. Especially where literacy levels are low, this may involve dramatizations, videos, road shows, etc. Whatever communication strategies are adopted, feedback should be sought from community members to ensure that the nature and purpose of the trial are adequately understood.

The rationale and justification for the trial must be explained clearly. So too must any risks involved, and the ways in which researchers propose to manage those risks (Section 7). Because no guarantee can be provided that risks can be completely avoided or averted, communities must be provided with a fair account of their probability and magnitude in order for them to provide authentic authorization for the trial to proceed. Answers should be provided to any widely held concerns. As evidence comes to light of local community (or broader societal) perceptions, concerns, or expectations, an effort should be made to respond appropriately.

Different stakeholders have different goals, and any trial description should acknowledge goals other than those of the researchers themselves. At least three types of goals should be recognized: overarching goals (e.g., to improve disease control, and thereby reduce disease-related mortality and morbidity); scientific goals (e.g., to test the efficacy of the GE strategy); collaborative researcher-community goals, likely to emerge from consultation with community members and an important component of maintaining local acceptance (e.g., improving local vector surveillance capacity)

\subsubsection{Get to know the community}

As a precondition to negotiating a mutually acceptable implementation strategy in any given setting, it is necessary to learn as much as practically possible about the trial site and the society in which it is located. Knowledge should include, but not be restricted to, familiarity with:

- Individuals and families located in the immediate vicinity of the proposed field cage

- The identity of key opinion leaders

- Any previous field trials of biological control or genetically engineered organisms, the legacy of which might influence attitudes towards the proposed trial

- Potential stakeholder organizations-that is, any organizations that are likely to consider that they have a "stake" in the trial's implementation, whether from a favorable or critical stance

- Mechanisms and levels of governance, and associated power structures (Section 8)

- Informal power structures and networks

- Commercial organizations that might have an interest in the trial's implementation (including those that might perceive the trial as threatening, such as tourist industry organizations who might not wish to publicize the presence of the disease in the region)

- Major ethnic and/or religious groups in the community, and relationships among them

- Key media organizations

- Significant economic, political, cultural, or other issues that have provided a basis for mobilization in the community in recent years

- Significant economic, social, and/or cultural assets that are valued by the community.

The knowledge gained should form part of the basis for making a final site selection decision. Once the decision about a field site has been made, further efforts should be undertaken in order to gain a more comprehensive understanding of local views, such as how the people who live in a particular area define the 
local community. This is best achieved through building relationships and conducting qualitative in-depth research, usually involving group discussions/focus groups. Surveys of local knowledge, attitudes, and practices (KAP) can provide an excellent way to gain insights from large numbers of people quickly. However KAP surveys usually require communities to respond to the conceptualizations of the researchers as articulated in the specific survey questions. These may be more valuable therefore if they follow in-depth qualitative research to discover the perspective of the local communities, which would facilitate development of the survey instrument. In learning about a local community, it is also important to be alert to groups or categories of people who may, in effect, be hidden because they do not have the power or status to gain attention or to express their own views. This may occur because of religious, ethnic, or gender differences.

\subsubsection{Understand the community's exposure to and experience of the disease(s) under investigation}

This will include familiarization with epidemiological and clinical data about disease outbreaks and the relevant disease control system(s). Among other reasons, this will help the researchers to establish whether their stated scientific justification and rationale for the trial are likely to resonate with the local communities. Further, this should include both quantitative and qualitative data gathering about people's:

- Exposure to/perceived risk of the disease under investigation

- Household and/or community practices for preventing and managing this disease

- Beliefs relating to the nature, causes, and consequences of this disease

- Beliefs relating to who shares responsibility for controlling this disease

- Perceptions regarding biological control and genetic engineering, including perceptions relating to risks

- Beliefs and attitudes regarding research and research-related risk

- Attitudes towards participation/involvement in research.
Gathering these data will require a mix of social science research methods, in particular:

- Using available official data about prevalence and incidence of the disease under investigation (and other key diseases)

- Conducting KAP or similar surveys

- Conducting in-depth interviews with key informants

- Forming focus groups, or some other culturally acceptable way of gathering qualitative data about people's perceptions and experiences.

\subsubsection{Establish relationships, build trust}

A community's willingness to support a project such as a caged field trial will depend largely on the extent to which researchers are able to foster relationships of trust and mutual respect. This takes time-and demands that researchers (and their funders) respect the tempo of local community processes as well as their own timetables. Researchers must find ways to make themselves available to the community, so that they can become known by community members. Demonstration that the project is providing local training and capacity building opportunities, or other valued services, can help to engender community goodwill.

Community support also requires a willingness to listen to a community's needs. Although many of these needs may lie beyond the capacity of researchers to address, there may be opportunities to incorporate local aspirations into the project. These should be taken seriously by researchers and by those agencies or organizations supporting the research. Researchers will need to work closely with the community to determine what will constitute a fair distribution of benefits in the context of the trial and ancillary studies.

\subsubsection{Secure the community's endorsement}

Implementation of a caged field trial will require some form of explicit endorsement by the community in which it takes place. The form that this endorsement should take, and the steps best taken to secure it, should themselves be determined in consultation with the community. 
Depending on the wishes of the community, the requirements of the relevant institutional ethics committees or review boards, and the laws of the host country, it also may be necessary to obtain some form of informed consent from individuals participating in the trial. If so, procedures for obtaining individual consent should also be discussed with, and be acceptable to, members of the community.

Community endorsement should be viewed, not as a one-time "green light" for the project to commence, but as an important ongoing manifestation of the relationship between the project and the local community.

\subsubsection{Establish means of representation and communication}

For these steps to occur, researchers need to identify and negotiate with a group or individuals whose claim to represent the community is seen as legitimate by other members of that community. In some instances, this might be a relatively straightforward matter, but in others-as when a community is riven by ethnically-based or other divisions-legitimacy might be contested. This may be particularly true in situations involving "nested" communities.

It is also necessary to put in place a mechanism-acceptable to all parties-for the ongoing representation of community interests and for channeling communication between parties. This might be an existing body, such as a community council, or the parties might prefer to set up a separate body. It would be valuable to have representation from the research team on this body, perhaps as an observer, in order to facilitate communication. Agreements regarding such issues as meeting arrangements and frequency, responsibilities for feedback, and communicating with the media, should all be clarified through negotiation as part of establishing acceptable means of representation.

\subsubsection{Maximize opportunities for community stewardship of the project}

Community support is unlikely to be secured-much less sustained-unless members of a community feel a sense of ownership, or stewardship, of the project. In recent decades, a literature has grown up around a number of concepts and models that seek to foster such a sense: community development, community control, empowerment, community ownership, to name a few. Many of these build on the seminal description of the "Ladder of Citizen Participation" (Arnstein 1969), a phrase coined originally in the context of attempts in the United States to foster public participation in planning decisions. Arnstein distinguished eight rungs, as shown in Figure 5. As Arnstein argued, all of the steps on the ladder have been used at various times to create the appearance of participation. Many attempts to implement "community ownership" can be (and have been) criticized as mechanisms whereby those with the power to set and control agendas seek to enlist compliance from those who have no such power by creating the illusion that power is being shared.

To avoid exposing the project to such charges, three principles should be followed:

- Community stewardship is an important factor in the success of community engagement approaches and must be taken seriously by researchers. This requires negotia-

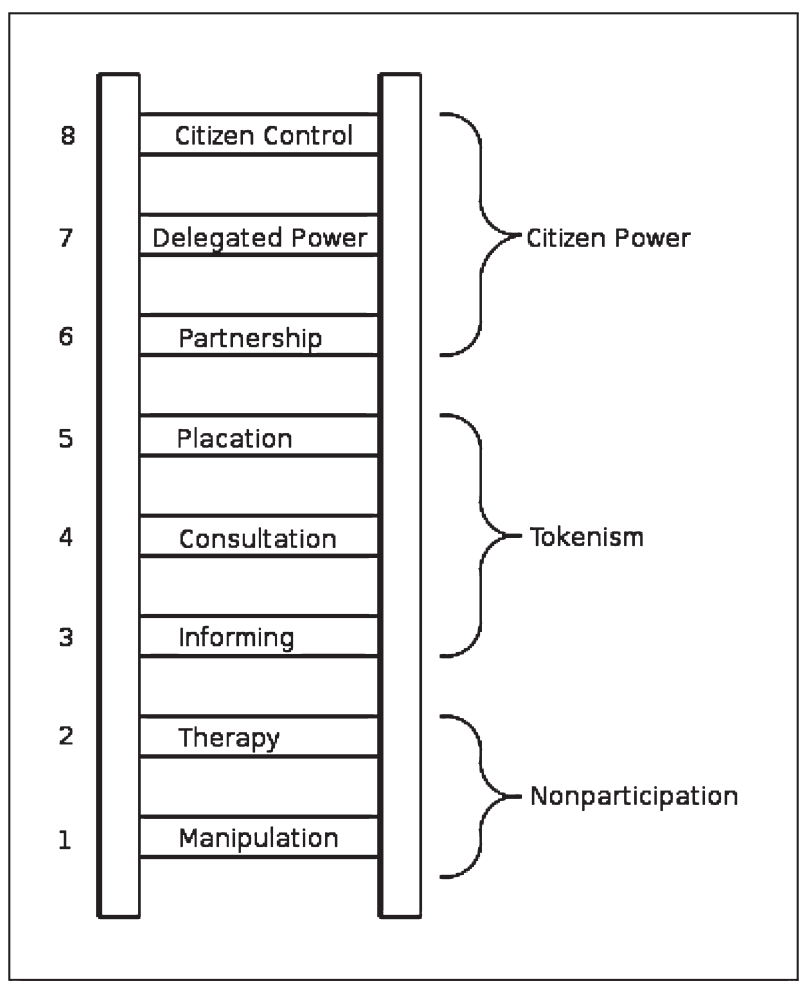

FIG. 5. Arnstein's Ladder of Participation, available online at http://lithgow-schmidt.dk/sherry-arnstein/ladderof-citizen-participation.html\#download. 
tion of clear understandings between the community and the project team about the rights and responsibilities of all parties involved in implementing the project.

- Researchers should be open to community wishes for a degree of control over the conduct of any trial that might lie beyond what they are accustomed to-especially if, by doing so, they demonstrate good faith with respect to community involvement.

- Researchers should respect-and listen todissenting opinions and priorities, and ensure that these are considered in discussions and decisions.

\subsection{A Note on Public Engagement}

Public engagement refers to interaction with the wider society in which the community of interest is embedded. This may include activities and processes undertaken by or on behalf of the researchers to gauge the level of support of the population for the trial, increase public understanding of the trial, or enlist public support for the trial. Whereas the processes of community engagement normally involve quite a lot of face-to-face interaction, public engagement will be conducted, in part at least, at a more impersonal level-for example, by developing a media strategy.

Strategies for engaging with the wider public - that is, with individuals and groups in the society beyond the actual trial site or sites-must be developed, and these ideally will complement community engagement activities. It is important to recognize the existence of different "publics" and tailor communications accordingly, taking account not only of the concerns of particular groups but also their likely level of scientific education.

As with community engagement, the principles here should include:

- Becoming informed about historical and cultural aspects of the society likely to influence views about caged field trials of GE mosquitoes

- Identifying key stakeholder groups, especially those likely to be alarmed about such trials
- Developing a proactive communication strategy, with the intention of informing key media outlets, journalists, politicians, etc.

- Monitoring public comment on the trial, and responding rapidly to any misinformation.

In the context of contained field trials for GE mosquitoes, it will be important to be proactive. This will require anticipating issues before they arise and formulating responses in advance, rather than waiting to respond to criticisms as they emerge. It also will be important that the project team speak "with one voice" in order to avoid disseminating a muddled message. Early development of a set of talking points, designed to get across key information in an audience-appropriate way and to address expected questions, may be helpful in formulating a unified communications strategy. Also in this regard, the research team should consider appointing one member as the spokesperson for the project.

\section{CONCLUSION}

The initiation of field trials to assess the safety and efficacy of "population replacement" genetic strategies for controlling vector-borne diseases will require extensive forward planning and preparation. Significant scientific challenges currently remain unsolved. Even so, recent scientific progress has been sufficiently robust to justify serious consideration of the practical issues and challenges that must be addressed in order to move forward. Further advancement of genetic control strategies will require the development of a complete technological pathway, from the construction of GE mosquitoes through their evaluation for safety and efficacy. This should be integrated with a site development pathway leading to approval and conduct of trials under circumstances relevant to the eventual introduction of the strategy into disease endemic regions (Fig. 6). This guidance document proposes a plan for phased testing, taking into account both safety concerns and scientific issues. It advocates a series of contained field trials that follow promising results in laboratory studies. It provides practical recommendations for field site selection, preparation for and conduct of such trials, including appropriate mea- 


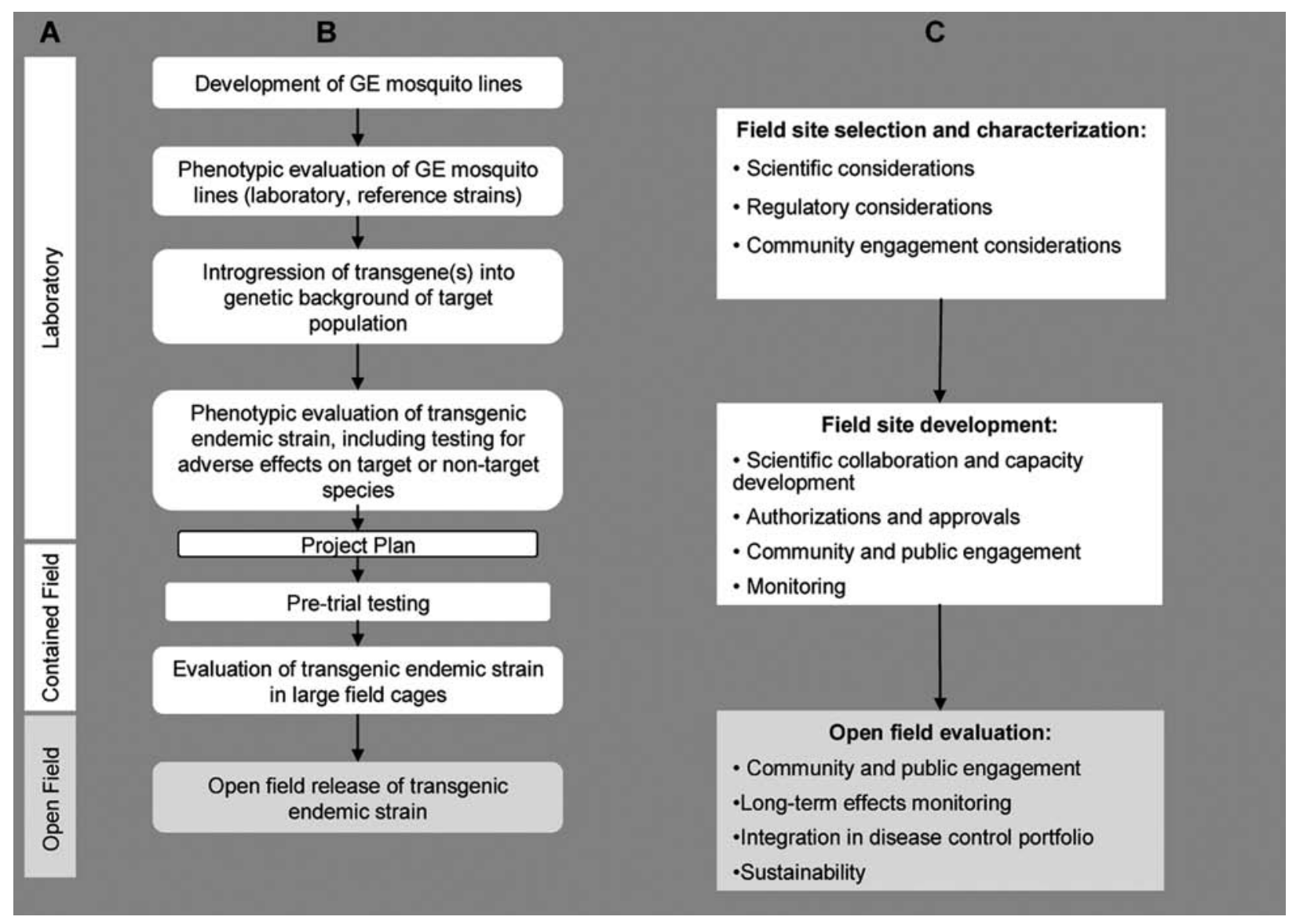

FIG. 6. Illustration of an integrated pathway for testing of GE mosquitoes (as defined in Section 2). Column A, testing phase; Column B, research and technology development pathway; Column C, field site development pathway. The figure is for guidance only, and the process must be adapted to fit the needs of the specific circumstances. Boxes shaded light gray would apply to open field testing, which is not addressed in this guidance document. (Adapted from Knols et al., 2007.)

sures to prevent unintended escape and to mitigate the consequences of such an escape in the unlikely event it should occur. Important regulatory and community engagement considerations also are discussed.

The Working Group purposely chose to focus these recommendations on the strategy of population replacement, employing GE mosquitoes containing a gene drive system (as defined in Section 2), in an effort to tackle the broadest range of issues and concerns arising from a genetic engineering approach. Not all of the precautions recommended here may be considered relevant to or necessary for other genetic strategies, such as those aimed at population reduction. The Working Group hoped, however, that by aiming for the most comprehensive and rigorous requirements this guidance will inform the planning process for con- tained field trials more generally by ensuring that important considerations will not be overlooked. This guidance does not extend to the testing of GE mosquitoes experimentally infected with a pathogen, nor does it extend to subsequent phases of the testing process beyond cage trials. It may be assumed that promising results in contained field trials would lead to discussion of possible open field trials. Additional guidance would need to be developed to progress to open field release of GE mosquitoes, building on the knowledge derived from contained trials and taking into account the additional complexities that will be involved.

The Working Group recognizes the need for additional deliberation on some of the important issues that are raised in these recommendations, such as requirements for risk assess- 
ment and community acceptance, and strongly encourages further dialogue on these topics. It is anticipated that the knowledge gained as this research advances likely will require that these recommendations be updated in the future. The recommendations for early phases of field testing provided within this guidance document are intended to stimulate the discussion that will be necessary to define a pathway for further assessment of the utility of this innovative approach for reducing or preventing the transmission of vector-borne diseases.

\section{ACKNOWLEDGMENTS}

The Working Group members wish to thank the following individuals for reviewing the draft recommendations and providing extremely valuable comments and suggestions: Gerard Barry, Wendy Fink, Stuart Pimm, Megan Quinlan, Michael Rodemeyer, Tony Shelton, and James Tiedje. Support for coordination of the Working Group and preparation of this document was provided by The Foundation for the National Institutes of Health through the Grand Challenges in Global Health initiative.

\section{REFERENCES}

Alphey, L, Beard, CB, Billingsley, P, Coetzee M, et al. Malaria control with genetically manipulated insect vectors. Science 2002; 298:119-121.

Arnstein, SR. A ladder of citizen participation. J Am Inst Planners 1969; 35:216-224.

Arthropod Containment Levels. Vector-Borne and Zoonotic Diseases 2003; 3: 75-90.

Bertolla, F, Simonet, P. Horizontal gene transfers in the environment: natural transformation as a putative process for gene transfers between transgenic plants and microorganisms. Res Microbiol 1999; 150:375-384.

Braig, HR, Yan, G. The spread of genetic constructs in natural populations. In: Lettourno, DK, Burrows, DK, eds. Boca Raton: CRC Press; 2002:251-314.

Collins, FH, James, AA. Genetic modifications of mosquitoes. Sci Med 1996; 3:52-61.

Curtis, CF, Grover, KK, Suguna, SG, Uppal, DK, et al. Comparative field cage tests of population suppressing efficiency of three genetic control systems for Aedes aegypti. Heredity 1976; 36:11-29.

Environmental Protection Agency. Guidelines for ecological risk assessment. EPA630/R-95-002F, Washington, DC, USA, 1998.
Fiksel, J, Covello, VT. Biotechnology risk assessment: issues and methods for environmental introductions. Pergamon Press; 1986.

Garrett-Jones, C, Shidrawi, GR. Malaria vectorial capacity of a population of Anopheles gambiae. Bull WHO 1969; 40:531-454.

Gould, F, Schliekelman, P. Population genetics of autocidal control and strain replacement. Ann Rev Entomol 2004; 49:193-217.

Hardy, JL, Houk, EJ, Kramer, LD, Reeves, WC. Intrinsic factors affecting vector competence of mosquitoes for arboviruses. Ann Rev Entomol 1983; 28:229-262.

Harrington, L. Dispersal of the dengue vector Aedes aegypti within and between rural communities. Am J Trop Med Hyg 2005; 72:209-230.

Hill, RA, Sendashonga, C. General principles for risk assessment of living modified organisms: lessons from chemical risk assessment. Envir Biosafety Res 2003; 2:81-88.

Hoy, MA. Evaluating potential risks of transgenic arthropods for pest management programmes. In: Status and Risk Assessment of the Use of Transgenic Arthropods in Plant Protection. Austria: IAEA-TECDOC-1483; 2006; 121-144.

Juma, C, Serageldin I. Freedom to Innovate: Biotechnology in Africa's Development, A report of the High-Level Panel on Modern Biotechnology. Addis Ababa and Pretoria: African Union and New Partnership for Africa's Development; 2007.

Knols, BG, Bossin, HC, Mukabana, WR, Robinson, AS. Transgenic mosquitoes and the fight against malaria: Managing technology push in a turbulent GMO world. Am J Trop Med Hyg 2007; 77(Suppl 6):232.

Knols, BGJ, Njiru, BN, Mukabana, RW, Mathenge, EM, et al. Contained semi-field environments for ecological studies on transgenic African malaria vectors: benefits and constraints. In: Ecological Aspects for Application of Genetically Modified Mosquitoes. Wageningen UR Frontis series 2002; 2:91-106.

Pew Initiative. Bugs in the System: Issues in the Science and Regulation of Genetically Modified Insects. Washington, DC, 2004.

American Committee of Medical Entomology: American Society of Tropical Medicine and Hygiene. Risk Assessment for Arthropod Vectors. Vector Borne Zoonot 2003; 3:69-73.

Scott, TW, Takken, W, Knols BGJ, Boete C. The ecology of genetically modified mosquitoes. Science 2002; 298:117-119.

Sinkins, SP, Gould, F. Gene drive systems for insect disease vectors. Nature Genet 2006; 7:427-435.

Snow, AA, Andow, DA, Gepts, P, Hallerman, EM, et al. Genetically engineered organisms and the environment: current status and recommendations. Ecolog Appl 2005; 15:377-404.

Toure, YT, Oduola, AMJ, Sommerfeld, J, Morel, CM. Biosafety and risk assessment in the use of genetically modified mosquitoes for disease control. In: Ecological Aspects for Application of Genetically Modified Mosquitoes. Wageningen UR Frontis series 2002; 2:217-222. 